Document downloaded from:

http://hdl.handle.net/10251/78709

This paper must be cited as:

Rueda, P.; Sánchez Pérez, EA. (2015). Factorization theorems for homogeneous maps on banach function spaces and approximation of compact operators. Mediterranean Journal of Mathematics. 12(1):89-115. doi:10.1007/s00009-014-0384-3 0378-620X/15/010089-27.

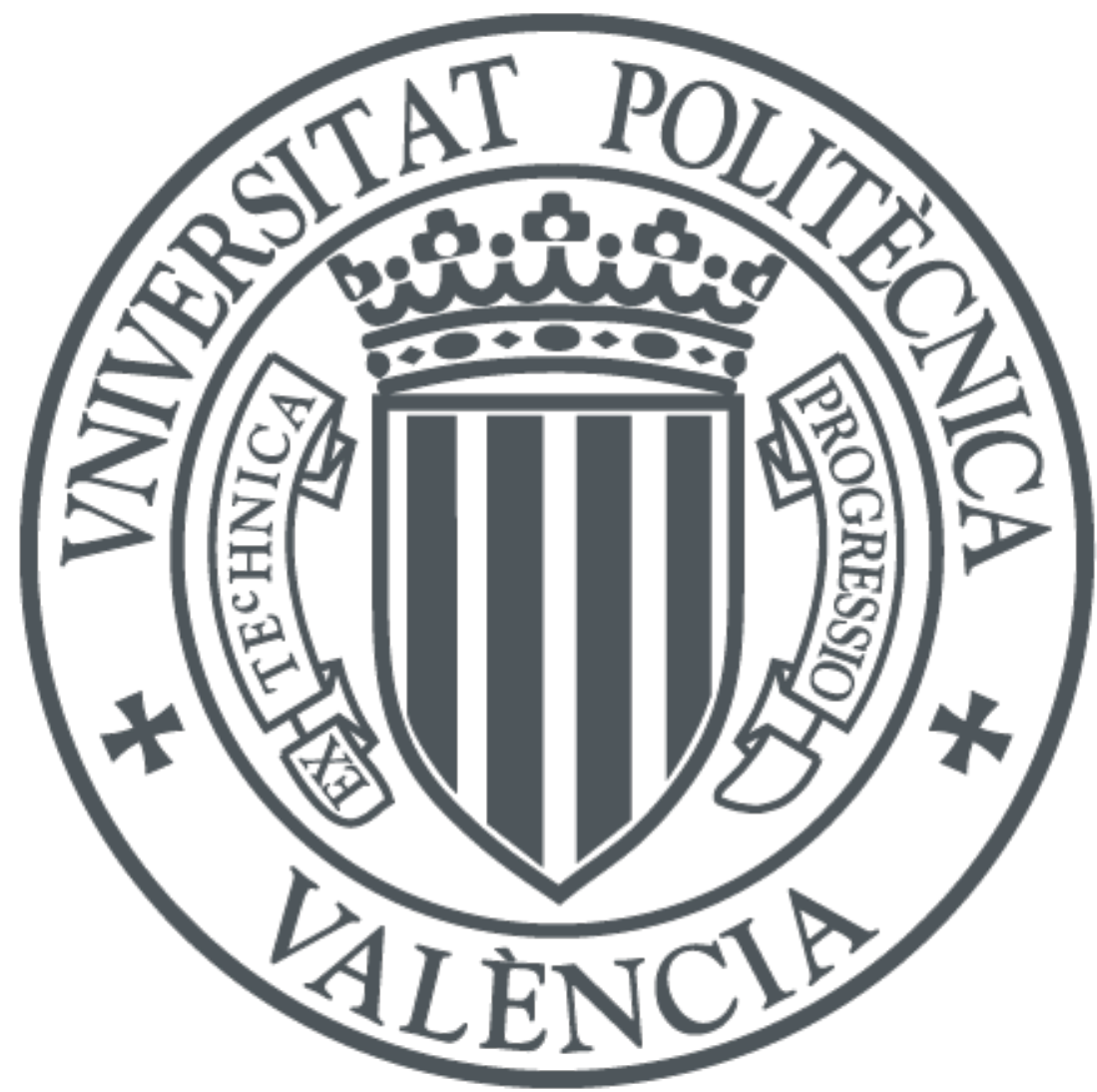

The final publication is available at

http://dx.doi.org/10.1007/s00009-014-0384-3

Copyright Springer Verlag (Germany)

Additional Information

The final publication is available at Springer via http://dx.doi.org/10.1007/s00009-014-0384-3 


\title{
FACTORIZATION THEOREMS FOR HOMOGENEOUS MAPS ON BANACH FUNCTION SPACES AND APPROXIMATION OF COMPACT OPERATORS
}

\author{
PILAR RUEDA AND ENRIQUE A. SÁNCHEZ-PÉREZ
}

\begin{abstract}
In this paper we characterize compact linear operators from Banach function spaces to Banach spaces by means of approximations with bounded homogeneous maps. To do so, we undertake a detailed study of such maps, proving a factorization theorem and paying special attention to the equivalent strong domination property involved. Some applications to compact maximal extensions of operators are also given.

Banach function space and $p$-th power and compact operator and homogeneous operator. 46E30 and 47B38 and 46B42 and 46B28
\end{abstract}

\section{INTRODUCTION}

It is well-known that compact linear operators between Banach spaces can be approximated by a sequence of "elementary maps" - finite range linear operators whenever the approximation property is considered. Here we are interested in compact linear operators defined on Banach function spaces into Banach spaces. We will see that, in this case, no approximation properties are required to get approximations by other elementary maps: bounded homogeneous maps with some nice properties. These properties are related to the way homogeneous maps can factor. As far as we know, there are no powerful classical factorization theorems for homogeneous operators others than the so called Maurey-Rosenthal theorems (see for example $[2,3]$ ). Therefore, we provide a factorization theorem for a class of homogeneous maps, that will give interesting information in our study of compact linear operators.

These considerations yield to two main purposes in this paper. On one hand to isolate those homogeneous maps that factor through a given homogeneous map and to characterize them by means of a strong domination property. On the other hand, to apply this study of strongly dominated homogeneous maps to characterize compact operators, from Banach function spaces into Banach spaces, as norm limits of homogeneous maps that satisfy a domination property. We prove that in some cases it is also possible to obtain a factorization of the compact operator itself. To get such characterizations, we consider those homogeneous maps that are dominated by a distinguished homogeneous map, the so-called $\phi_{p}$. This map will allow to describe order bounded sets, that play an important role in the study of compact operators. Some applications to operators allowing a compact extension to their optimal domain -i.e. to the biggest Banach function space to which they can be extended - are also given. Our most basic ideas are inspired in a classical technique that has its roots in the Grothendieck's description of weakly compact sets. The

Date: Received: date / Accepted: date. 
fact of considering the setting of Banach function spaces allows to combine these techniques with tools as $p$-convexifications or Maurey-Rosenthal type theorems.

The paper is organized as follows. In Section 1 we fix notation and review definitions and basic properties on Banach functions spaces. In Section 2 we introduce strongly dominated homogeneous maps and characterize them by means of a factorization scheme. We define the distinguished homogeneous map $\phi_{p}: X(\mu) \rightarrow X_{[1 / p]}$, where $X(\mu)$ is a Banach function space and $X_{[1 / p]}$ is its $p$-convexification, by $\phi_{p}(\cdot):=(\cdot)^{1 / p}\|(\cdot)\|^{1 / p^{\prime}}$ and use it to characterize order bounded sets in $X$. In particular, homogeneous maps that are strongly dominated by $\phi_{p}$ take the unit ball of $X$ to a uniformly $\mu$-absolutely continuous set. A weaker domination by $\phi_{p}$ described as a norm inequality (see Proposition 2), is also characterized by means of a factorization property, and yields to a property closely related to uniformly $\mu$-absolutely continuity. This property transforms essentially compact operators to compact operators as shown in next section (Proposition 3). In Section 3 we develop the consequences of the previous factorization/domination results to the study of compact linear operators $T: X(\mu) \rightarrow E$, where $X(\mu)$ is a Banach function space and $E$ is a Banach space. The main characterizations of compact operators are included in Theorem 3. Finally, in Section 4 we give some applications to characterize compact optimal extensions of (compact) operators.

\section{BACKGROUND AND NOTATION}

Our notation is standard. If $E$ is a Banach space, $B_{E}$ and $S_{E}$ are the closed unit ball and the unit sphere, respectively. We will write $E^{*}$ for its topological dual. If $\phi: U \rightarrow E$ is a homogeneous map -i.e. a map that satisfies $\phi(\lambda x)=\lambda \phi(x)$, for all $x \in U$ and all $\lambda \geq 0$ - from a homogeneous subset $U$ of a Banach space $F$, we will call $\|\phi\|:=\sup _{x \in U \cap B_{F}}\|\phi(x)\|$ the norm of $\phi$ on $U$. Convergence of sequences of homogeneous maps will be considered in the sense of convergence with respect to this norm. Given $1 \leq p<\infty$, we write $p^{\prime}$ for the conjugate of $p$, i.e. $\frac{1}{p}+\frac{1}{p^{\prime}}=1$. Along the paper, $(\Omega, \Sigma, \mu)$ denotes a finite measure space, and $L^{0}(\mu)$ the space of equivalence classes of $\mu$-measurable functions. Following the definition in $[7$, Def.1.b.17, p.28], we say that a real Banach space $X(\mu)$ of (equivalence classes of) $\mu$-measurable functions in $L^{0}(\mu)$ is a Banach function space (also called Köthe function space) over $\mu$ if $X(\mu) \subset L^{1}(\mu)$ and contains all the characteristic functions of measurable sets, and the norm $\|\cdot\|_{X(\mu)}$ satisfies that if $f \in L^{0}(\mu), g \in X(\mu)$ and $|f| \leq|g| \mu$-a.e. then $f \in X(\mu)$ and $\|f\|_{X(\mu)} \leq\|g\|_{X(\mu)}$. The inclusions $L^{\infty}(\mu) \subset X(\mu) \subset L^{1}(\mu)$ are always continuous, since they are positive. We will write $X$ instead of $X(\mu)$ if the measure is clear in the context. A Banach function space $X$ is order continuous if increasing sequences that are bounded $\mu$-a.e. are convergent in norm. If $X(\mu)$ and $Z(\mu)$ are Banach function spaces and $X \subseteq Z$, we define the space of multiplication operators $X^{Z}$ as the space of (classes of) measurable functions defining operators from $X$ to $Z$ by pointwise multiplication, endowed with the operator norm. It is also a Banach function space over $\mu$. This space can also be defined between Banach function spaces $X(\nu)$ and $X(\mu)$, when the measures $\nu$ and $\mu$ are defined on the same $\sigma$-algebra and are equivalent.

A relevant space of multiplication operators is given by $X^{L^{1}(\mu)}$, the Köthe dual of $X(\mu)$, also called the associate space. It is the order ideal of $L^{0}(\mu)$ defined by

$$
X(\mu)^{\prime}:=\left\{g \in L^{0}(\mu): g \cdot X(\mu) \subset L^{1}(\mu)\right\} .
$$


Each $g \in X(\mu)^{\prime}$ can be identified with a continuous functional on $X(\mu)$ via the integral of the multiplication operator $f \in X(\mu) \mapsto f g \in L^{1}(\mu)$.

If $\Omega=[0,1]$, we always consider Lebesgue measure on the space. For $1 \leq r<\infty$, we write $L^{r}[0,1]$ for the Lebesgue space of $r$-integrable functions.

Let $0<p<\infty$. If $f$ is a measurable function, we write $f^{1 / p}$ for the measurable function $\operatorname{sign}\{f\}|f|^{1 / p}$. If $X(\mu)$ is a Banach function space, the $p$-convexification of $X$ is defined as

$$
X_{[p]}:=\left\{f \in L^{0}(\mu):|f|^{1 / p} \in X\right\}
$$

that is a quasi-Banach function space over $\mu$ when endowed with the seminorm $\|f\|_{X_{[p]}}:=\left\||f|^{1 / p}\right\|_{X}^{p}, f \in X_{[p]}$. It is a Banach space and the above expression defines a norm if and only if $X$ is $p$-convex with $p$-convexity constant 1 . For instance, $L^{p}[0,1]_{[p]}=L^{1}[0,1]$ and $L^{1}[0,1]_{[1 / p]}=L^{p}[0,1]$ isometrically. It is important to note that every Banach function space $X(\mu)$ is $r$-convex (with $r$-convexity constant equal to one) if $0<r<1$, and so $X_{[r]}$ is always a Banach function space with the norm given above. Other important fact that can be easily verified is that for $1 \leq p<\infty$ and for each $f \in X(\mu)_{[1 / p]}$, its norm can be computed as $\|f\|_{X_{[1 / p]}}:=$ $\sup _{h \in B_{X_{\left[1 / p^{\prime}\right]}}}\|f h\|_{X}$. Moreover, the space of multiplication operators $\left(X_{[1 / p]}\right)^{X}$ from $X_{[1 / p]}$ to $X$ is the Fatou completion $\bar{X}_{\left[1 / p^{\prime}\right]}$ of $X_{\left[1 / p^{\prime}\right]}$ (see this definition under the name of maximal normed extension and also Theorem 5 in [8]). We say that a homogeneous subset $U$ of a Banach function space $X$ is solid if for every $f \in U$ and $A \in \Sigma, f \chi_{A} \in U$.

Let $E$ be a real Banach space and let $(\Omega, \Sigma)$ be a measurable space. A vector measure from $\Sigma$ into $E$ is a set function $m: \Sigma \rightarrow E$ which is countably additive. In particular, we are interested in vector measures defined by operators: given an operator $T: X(\mu) \rightarrow E$, we write $m_{T}$ for the vector measure given by $m_{T}(A):=$ $T\left(\chi_{A}\right)$ for $A \in \Sigma$. If $m: \Sigma \rightarrow E$ is a vector measure, the variation $|m|$ of $m$ on a measurable set $A$ is defined by $|m|(A):=\sup \sum_{B \in \Pi}\|m(B)\|$ for $A \in \Sigma$, where the supremum is computed over all finite measurable partitions $\Pi$ of $A$. It defines a (non necessarily finite) monotone countably additive function on $\Sigma$, i.e. a positive scalar measure. The semivariation of $m$ is defined by $\|m\|(A):=\sup _{B \subset A}\|m(B)\|$, $A, B \in \Sigma$. A set $A \in \Sigma$ is $m$-null if $\|m\|(A)=0$. An operator $T: X(\mu) \rightarrow E$ is said to be $\mu$-determined if each $m_{T}$-null set is $\mu$-null.

A $\Sigma$-measurable function $f$ is scalarly integrable with respect to $m$ (scalarly $m$ integrable) if it is integrable with respect to each scalar measure $\left\langle m, x^{\prime}\right\rangle$ given by $\left\langle m, x^{\prime}\right\rangle(\cdot):=\left\langle m(\cdot), x^{\prime}\right\rangle$. If besides for every $A \in \Sigma$ there is an element $\int_{A} f d m \in E$ such that $\left\langle\int_{A} f d m, x^{\prime}\right\rangle=\int_{A} f d\left\langle m, x^{\prime}\right\rangle, x^{\prime} \in E^{\prime}$, then it is said that $f$ is integrable with respect to $m$ ( $m$-integrable). The set of all the (classes of $m$-a.e. equal) scalarly $m$-integrable functions $L_{w}^{1}(m)$ defines a Banach function space with the Fatou property and with weak unit $\chi_{\Omega}$ —in the sense of [7, Def.1.b.17] — over any Rybakov measure $\nu=\left|\left\langle m, x_{0}^{\prime}\right\rangle\right|$ for $m$ (see [4, Ch.IX,2]) with the norm

$$
\|f\|_{L_{w}^{1}(m)}:=\sup _{x^{\prime} \in B_{E^{\prime}}} \int_{\Omega}|f| d\left|\left\langle m, x^{\prime}\right\rangle\right|, \quad f \in L_{w}^{1}(m) .
$$

The space $L^{1}(m)$ of (classes of) $m$-integrable functions is a Banach function subspace of $L_{w}^{1}(m)$ - with norm $\|\cdot\|_{L^{1}(m)}=\|\cdot\|_{L_{w}^{1}(m)}$-, and it is always order continuous and has a weak unit. The formula $\sup _{A \in \Sigma}\left\|\int_{A} f d m\right\|, f \in L^{1}(m)$, gives an equivalent expression of the norm in this space. Recall that a Rybakov measure 
has the same null sets that $m$. The reader can find the definitions and fundamental results concerning the space $L^{1}(m)$ and $L_{w}^{1}(m)$ in [12] and the references therein.

If $1<p<\infty$, the spaces $L^{p}(m)$ and $L_{w}^{p}(m)$ are defined extending the definition above in the natural manner. In fact, they can be defined as the $1 / p$-convexifications of $L^{1}(m)$ and $L_{w}^{1}(m)$, i.e. $L^{p}(m):=L^{1}(m)_{[1 / p]}$ and $L_{w}^{p}(m):=L_{w}^{1}(m)_{[1 / p]}$, respectively. In particular, they are $p$-convex Banach function spaces with weak unit $\chi_{\Omega}$ over a Rybakov measure, with the norm

$$
\|f\|_{L^{p}(m)}:=\left\||f|^{p}\right\|_{L^{1}(m)}^{\frac{1}{p}}, \quad f \in L^{p}(m) .
$$

Again, $L^{p}(m)$ is order continuous and $L_{w}^{p}(m)$ has the Fatou property. It can be easily seen that $L^{p}(m) \subseteq L^{1}(m)$. The reader can find more information on these spaces in $[5,6,12,13]$. Two interesting facts that will be used in the paper are that the space of multiplication operators from $L^{p}(m)$ to $L^{1}(m)$ is $L_{w}^{p^{\prime}}(m)$, and

$$
\|f\|_{L_{w}^{p}(m)}=\sup _{g \in B_{L^{p^{\prime}(m)}}}\left\|\int f g d m\right\|_{E}=\sup _{g \in B_{L^{p^{\prime}(m)}}}\|f g\|_{L^{1}(m)}, \quad f \in L_{w}^{p}(m),
$$

(see [12, Ch.3]). The expression $\sup _{A \in \Sigma}\left\|\int_{A}|f|^{p} d m\right\|^{1 / p}$ provides an equivalent norm.

We will consider the integration operator $I_{m}: L^{1}(m) \rightarrow E$ given by $I_{m}(f):=$ $\int_{\Omega} f d m, f \in L^{1}(m)$, and its restriction to $L^{p}(m)$, for which the same symbol will be used. The properties of the integration map has been largely studied in several papers (see $[11,12]$ and the references therein). We will be interested in spaces $L^{1}\left(m_{T}\right)$ for vector measures defined by operators $T$ from order continuous Banach function space $X(\mu)$ to a Banach space $E$ : it is well known that $L^{1}\left(m_{T}\right)$ represents the optimal domain for $T$, i.e. the greatest order continuous Banach function space to which the operator can be extended.

The terminology regarding order properties and compactness properties of operators on Banach lattices is variate and often confusing. So let us finish this section by establishing it clearly. We will deal mainly with two classes of (homogeneous norm bounded) operators $T$ from an order continuous Banach function space $X(\mu)$ with a weak unit - $\mu$ being finite - to a Banach function space $Z(\nu)$ or to a Banach space $E$.

1) A set $B \subset Z(\nu)$ is approximately order bounded if for each $\varepsilon>0$ there is $f \in Z(\nu)$ such that $B \subseteq[-f, f]+\varepsilon B_{Z}$. A (bounded homogeneous) operator $T: X(\mu) \rightarrow Z(\nu)$ is semicompact if $T\left(B_{X}\right)$ is approximately order bounded (see Section 3.6 and Definition 3.6.9 in [9]). A set $B \subseteq Z(\nu)$ is uniformly $\nu$-absolutely continuous if

$$
\lim _{\nu(A) \rightarrow 0} \sup _{f \in B}\left\|f \chi_{A}\right\|_{Z}=0
$$

(see Section 2 and the comments around Lemma 2.37 in [12]). These sets are also sometimes called uniformly $\mu$-integrable sets. We will deal with maps $T$ : $X(\mu) \rightarrow Z(\nu)$ that satisfy that $T\left(B_{X}\right)$ is a uniformly $\nu$-absolutely continuous subset of $Z$, and call them uniformly $\nu$-absolutely continuous maps. For the case of order continuous Banach function spaces, the class of uniformly $\nu$-absolutely continuous operators and semicompact operators coincide (see Lemma 2.37 in [12]).

2) A continuous linear operator $T: X(\mu) \rightarrow E$ is said to be essentially compact if the set $\left\{T\left(\chi_{A}\right): A \in \Sigma\right\}$ is relatively (norm) compact in $E$. If $X(\mu)$ is order continuous and $m_{T}$ is the vector measure associated to $T$, this is equivalent to the range of $m_{T}$ 
being relatively compact. Trivially, if $m$ is a vector measure then the integration operator $I_{m}$ is essentially compact if and only if the range of $m$ is relatively compact. It is easy to see that essential compactness of an operator $T$ is equivalent to the fact that the restriction of $T$ to the space $L^{\infty}(\mu)$ is compact; in fact, the arguments on the paper uses often that for order continuous Banach function spaces this is equivalent to the operator being AM-compact in the terminology of [9], i.e. T maps order bounded sets to relatively compact sets (see Section 3.7 in [9]).

\section{A DOMINATION THEOREM FOR HOMOGENEOUS MAPS}

In this section we introduce a strong domination property between homogeneous operators and its equivalent formulation in terms of a factorization. This factorization theorem for strong dominated homogeneous operators is based in a separation argument that has shown to be the key for proving factorization theorems for operators between Banach function spaces. Similar arguments for the case of the factorization of linear operators have been recently developed in [3] (see also [2]). In what follow we show that they also work for the case of homogeneous operators. Whenever a homogeneous map $S: X \rightarrow X$ can factorize through $X_{[1 / p]}$ then bounded sets of $X$ are mapped by $S$ to approximately order bounded sets. This will allow us to characterize compactness of linear operators. First let us recall Ky Fan's lemma (see e.g. [12, Lemma 6.12]).

Ky Fan's lemma. Let $W$ be a compact convex subset of a Hausdorff topological vector space and let $\Psi$ be a concave family of lower semi-continuous, convex, $\mathbb{R}$ valued functions on $W$. Let $c \in \mathbb{R}$. Suppose, for every $\psi \in \Psi$, that there exists $x_{\psi} \in W$ such that $\psi\left(x_{\psi}\right) \leq c$. Then there exists $x \in W$ such that $\psi(x) \leq c$ for all $\psi \in \Psi$.

Let $E$ be a Banach space, and let $Y(\nu)$ and $Z(\nu)$ be Banach function spaces such that $Y(\nu) \subseteq Z(\nu)$. Let $U \subseteq E$ be a homogeneous set, and $\phi: U \rightarrow Y(\nu)$ and $P: U \rightarrow Z(\nu)$ be bounded homogeneous maps. Assume also that $Y^{Z}$ has the Fatou property and $\left(Y^{Z}\right)^{\prime}$ is order continuous. Then, for $K>0$, the following statements are equivalent.

(i) For every $x_{1}, \ldots, x_{n} \in U$ and $A_{1}, \ldots, A_{n} \in \Sigma$,

$$
\left\|\sum_{i=1}^{n}\left|P\left(x_{i}\right)\right| \chi_{A_{i}}\right\|_{L^{1}(\nu)} \leq K\left\|\sum_{i=1}^{n}\left|\phi\left(x_{i}\right)\right| \chi_{A_{i}}\right\|_{\left(Y^{Z}\right)^{\prime}} .
$$

(ii) There is a function $g \in K B_{Y z}$ such that

$$
|P(x)| \leq g|\phi(x)| \quad \mu-a . e .
$$

(iii) There is a function $g \in K B_{Y^{Z}}$ such that for every $x \in U$ there is a function $h_{x} \in B_{L^{\infty}(\nu)}$ depending only on $x /\|x\|$ such that $P(x)=g \cdot h_{x} \cdot \phi(x)$. In other words, $P$ factorizes through the homogeneous map $\hat{\phi}$ given by $\hat{\phi}(x):=h_{x} \cdot \phi(x)$ as

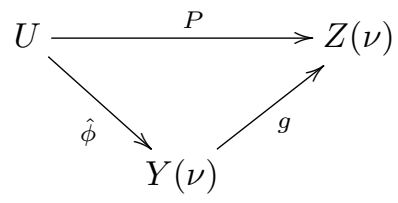

In this case we will say that $P$ is strongly dominated by $\phi$. 
Proof. Since $Y^{Z}$ has the Fatou property and $\left(Y^{Z}\right)^{\prime}$ is order continuous, we have that $Y^{Z}=\left(Y^{Z}\right)^{\prime \prime}=\left(\left(Y^{Z}\right)^{\prime}\right)^{*}$ (see [7, p.28-30]). Suppose (i) holds. For every finite sequence $x_{1}, \ldots x_{n} \in U$ and $A_{1}, \ldots, A_{n} \in \Sigma$, we define the convex function $\psi: B_{Y} Z \rightarrow \mathbb{R}$ by

$$
\psi(g)=\sum_{i=1}^{n} \int\left|P\left(x_{i}\right)\right| \chi_{A_{i}} d \nu-K \sum_{i=1}^{n} \int g\left|\phi\left(x_{i}\right)\right| \chi_{A_{i}} d \nu,
$$

for all $g \in B_{Y^{Z}} . \psi$ is continuous for the weak* topology on the compact convex set $B_{Y^{z}}$. Let $\mathcal{F}$ be the concave family of all these functions $\psi$. If $\psi \in \mathcal{F}$ is defined by means of $x_{1}, \ldots x_{n} \in X_{1}$ and $A_{1}, \ldots, A_{n} \in \Sigma$, take $g_{\psi} \in B_{Y^{z}}$ in such a way that

$$
\left\|\sum_{i=1}^{n}\left|\phi\left(x_{i}\right) \chi_{A_{i}}\right|\right\|_{\left(Y^{Z}\right)^{\prime}}=\int g_{\psi} \sum_{i=1}^{n}\left|\phi\left(x_{i}\right) \chi_{A_{i}}\right| d \nu .
$$

This element can be assumed to belong to the positive cone. This implies, by $(i)$, that $\psi\left(g_{\psi}\right) \leq 0$. By Ky Fan's lemma, there exists a function $g_{0} \in B_{Y^{z}}$ such that $\psi\left(g_{0}\right) \leq 0$ for all $\psi \in \mathcal{F}$. This means that

$$
\int_{A}|P(x)| d \nu \leq K \int_{A} g_{0}|\phi(x)| d \nu
$$

for every $x \in U$ and $A \in \Sigma$. This clearly implies that

$$
|P(x)| \leq K g_{0}|\phi(x)| \quad \mu-a . e .
$$

and (ii) is proved.

If we assume (ii), there is a function $h_{x} \in B_{L^{\infty}(\nu)}$ such that $P(x)=K h_{x} g_{0} \phi(x)$. It only rests to note that since $P$ and $\phi$ are homogeneous,

$$
P(x)=K\|x\| h_{x /\|x\|} g_{0} \phi(x /\|x\|)
$$

and so by writing $\hat{\phi}(x)=h_{x /\|x\|} \phi(x)$ and $g=K g_{0}$, we obtain the desired factorization through the homogeneous operator $\hat{\phi}$ given in (iii).

A direct calculation gives (iii) implies (i).

Take $U=X(\mu)=Z$ and $Y=X_{[1 / p]}$ for $1<p<\infty$. Let $0 \leq h \in X_{[1 / p]}$. The homogeneous map $P: X \rightarrow X$ given by $P(f):=\operatorname{sgn}(f)\|f\|\left(\frac{|f|}{\|f\|} \wedge h\right), f \in X$, is an example of a map satisfying (iii) in Theorem 2 , since it factors as $P=i_{p} \circ \phi$, for $\phi=P$, where $i_{p}: X_{[1 / p]} \rightarrow X$ is the inclusion map.

In the sequel, we will center our attention in the case $U=E=X(\mu), Z(\nu)=$ $X(\mu)$ and $Y(\nu)=X(\mu)_{[1 / p]}$ for a fixed $1<p<\infty$.

Recall that the space of multiplication operators $X_{[1 / p]} \rightarrow X$ coincides with the Fatou completion of $X_{\left[1 / p^{\prime}\right]}$, that we denote by $\overline{X_{\left[1 / p^{\prime}\right]}}$. In the case that $X$ has the Fatou property, then $X_{\left[1 / p^{\prime}\right]}$ has also the Fatou property. Thus, we have that $\overline{X_{\left[1 / p^{\prime}\right]}}=X_{\left[1 / p^{\prime}\right]}$ (see [8, p.325]). Therefore, $\left(X_{[1 / p]}\right)^{X}=X_{\left[1 / p^{\prime}\right]}$. Besides, if $X$ is order continuous then $X_{\left[1 / p^{\prime}\right]}$ is $p^{\prime}$-convex and so $\left(X_{\left[1 / p^{\prime}\right]}\right)^{\prime}$ is $p$-concave, this dual space is also order continuous. Thus, $X_{\left[1 / p^{\prime}\right]}$ has the Fatou property and so $X_{\left[1 / p^{\prime}\right]}=\overline{X_{\left[1 / p^{\prime}\right]}}=\left(X_{\left[1 / p^{\prime}\right]}\right)^{\prime \prime}=\left(\left(X_{\left[1 / p^{\prime}\right]}\right)^{\prime}\right)^{*}$. Therefore, the hypotheses of Theorem 2 are satisfied for order continuous Banach function space with the Fatou property.

Let $X(\mu)$ be an order continuous Banach function space with the Fatou property and $1<p<\infty$. Let $P: X(\mu) \rightarrow X(\mu)$ and $\phi: X(\mu) \rightarrow X(\mu)_{[1 / p]}$ be bounded 
homogeneous maps. If $P$ is strongly dominated by $\phi$ then $P\left(B_{X}\right)$ is a uniformly $\mu$-absolutely continuous set.

Proof. By Remark 2 the space of multiplication operators $X_{[1 / p]} \rightarrow X$ fulfills the conditions required in Theorem 2. By Theorem 2(ii), there is a function $g \in X_{\left[1 / p^{\prime}\right]}$ such that $|P(f)| \leq g|\phi(f)|, f \in X(\mu)$. Consequently, for each $A \in \Sigma$ and $f \in B_{X}$,

$$
\left\|P(f) \chi_{A}\right\| \leq\left\|g \chi_{A} \phi(f)\right\| \leq\left\|g \chi_{A}\right\|_{X_{\left[1 / p^{\prime}\right]}}\|\phi(f)\|_{X_{[1 / p]}} \leq\left\|g \chi_{A}\right\|_{X_{\left[1 / p^{\prime}\right]}}\|\phi\| .
$$

The order continuity of $X_{\left[1 / p^{\prime}\right]}$ gives the result.

Let $1<p<\infty$. A relevant particular case of homogeneous operator is the map $\phi_{p}: X(\mu) \rightarrow X(\mu)_{[1 / p]}$ given by

$$
\phi_{p}(f):=f^{1 / p}\|f\|_{X}^{1 / p^{\prime}}, \quad f \in X(\mu) .
$$

In this case, Proposition 2 can be refined in order to give a characterization of order bounded sets in $X(\mu)$. Recall that a bounded subset $B$ in the order continuous space $X(\mu)$ is uniformly $\mu$-absolutely continuous if and only if for every $\varepsilon>0$ there is a function $g_{\varepsilon} \in X(\mu)^{+}$such that $B \subseteq\left[-g_{\varepsilon}, g_{\varepsilon}\right]+\varepsilon B_{X}$, i.e. it is approximately order bounded (see for instance Lemma 2.37(iii) in [12]).

If $B \subseteq X(\mu)$ is a homogeneous set, we call the homogeneous operator $\phi_{B}$ : $X \rightarrow X$ given by $\phi_{B}(f):=f$ if $f$ belongs to $B$ and $\phi_{B}(f):=0$ otherwise, the characteristic homogeneous operator of $B$. We say that a homogeneous set $B \subset$ $X(\mu)$ is order bounded if $B \cap B_{X}$ is order bounded.

Let $X(\mu)$ be an order continuous space with the Fatou property. Let $1<p<\infty$. An homogeneous set $B \in X(\mu)$ is order bounded if and only if its characteristic homogeneous operator $\phi_{B}$ is strongly dominated by $\phi_{p}$.

Proof. Suppose that the characteristic homogeneous operator of $B$ is strongly dominated by $\phi_{p}$. Then by Theorem 2(ii) and Remark 2 there is a function $g \in X_{\left[1 / p^{\prime}\right]}$ such that $\left|\phi_{B}(\cdot)\right| \leq g\left|\phi_{p}(\cdot)\right|$. Then for every function $f$ in $B$ we have that

$$
|f(\cdot)| \leq g|f|^{1 / p} \cdot\|f\|_{X}^{1 / p^{\prime}} .
$$

Thus, $|f|^{1 / p^{\prime}}=|f| /|f|^{1 / p} \leq g\|f\|_{X}^{1 / p^{\prime}}$, and therefore $|f| \leq g^{p^{\prime}}\|f\|_{X}$. Since $g^{p^{\prime}} \in X$, this gives the result.

For the converse, let $h$ be the order bound for $B \cap B_{X}$. For every norm one element $f_{0}$ of $B,\left|f_{0}\right|^{1 / p^{\prime}} \leq h^{1 / p^{\prime}}$ and so for every $f \in B,(|f| /\|f\|)^{1 / p^{\prime}} \leq h^{1 / p^{\prime}}$ which gives the strong domination

$$
|f|=|f|^{1 / p}|f|^{1 / p^{\prime}} \leq|f|^{1 / p}\|f\|^{1 / p^{\prime}} h^{1 / p^{\prime}}
$$

of $\phi_{B}$ by $\phi_{p}$.

Let $\phi: X \rightarrow X$ be a bounded homogeneous map defined from an order continuous Banach function space with the Fatou property $X(\mu)$ such that the norm of the inclusion $X \hookrightarrow L^{1}(\mu)$ is equal to one. Suppose that $\|\phi\|=1$ and it satisfies that $\phi\left(|f|^{1 / p} \chi_{A}\right)=|\phi(f)|^{1 / p} \chi_{A}, f \in X, A \in \Sigma$. For instance, let $f \in X_{[1 / p]}, f \geq \chi_{\Omega}$, and consider

$$
U_{f}=\left\{g \in L^{0}(\mu) \text { : there exists } K>0 \text { so that }|g| \leq K f^{p}\right\} .
$$

Note that $|g|^{1 / p} \in U_{f}$ whenever $g \in U_{f}$ and that $L^{\infty}(\mu)=U_{\chi_{\Omega}}$. 
Since $X$ is order continuous and has the Fatou property, we have that $\left(X_{[1 / p]}\right)^{X}=$ $X_{\left[1 / p^{\prime}\right]}$ and $\left(\left(X_{\left[1 / p^{\prime}\right]}\right)^{\prime}\right)^{*}=X_{\left[1 / p^{\prime}\right]}$ (see Remark 2). Assume that $\phi$ is also well defined and positively 1-summing when considered as a function $\phi:\left(X_{\left[1 / p^{\prime}\right]}\right)^{\prime} \rightarrow L^{p}(\mu)$, that is, there is a constant $K>0$ such that for all positive elements $g_{1}, \ldots g_{n} \in X$,

$$
\sum_{i=1}^{n}\left\|\phi\left(g_{i}\right)\right\|_{L^{p}(\mu)} \leq K \sup _{h \in B_{X_{\left[1 / p^{\prime}\right]}}} \sum_{i=1}^{n}\left|\left\langle g_{i}, h\right\rangle\right| .
$$

In this case, for all $f_{1}, \ldots, f_{n} \in X$,

$$
\begin{aligned}
& \left\|\sum_{i=1}^{n}\left|\phi\left(f_{i}\right)\right|\right\|_{L^{1}(\mu)}=\sum_{i=1}^{n}\left\|\phi\left(f_{i}\right)\right\|_{L^{1}(\mu)} \leq \sum_{i=1}^{n}\left\|\phi\left(f_{i}\right)^{1 / p}\right\|_{L^{p}(\mu)}\left\|\phi\left(f_{i}\right)\right\|_{L^{1}(\mu)}^{1 / p^{\prime}} \\
\leq & \sum_{i=1}^{n}\left\|\phi\left(\left|f_{i}\right|^{1 / p}\right)\right\|_{L^{p}(\mu)}\|\phi\|^{1 / p^{\prime}}\left\|f_{i}\right\|_{X}^{1 / p^{\prime}} \leq K^{\prime} \sup _{h \in B_{X_{\left[1 / p^{\prime}\right]}}} \sum_{i=1}^{n}\left|\left\langle\left|f_{i}\right|^{1 / p}, h\right\rangle\right|\left\|f_{i}\right\|_{X}^{1 / p^{\prime}} \\
\leq & K^{\prime} \sup _{h \in B_{X_{\left[1 / p^{\prime}\right]}}} \int\left(\sum_{i=1}^{n}\left|f_{i}\right|^{1 / p}\left\|f_{i}\right\|_{X}^{1 / p^{\prime}}\right)|h| d \mu \leq K^{\prime}\left\|\sum_{i=1}^{n}\left|f_{i}\right|^{1 / p}\right\| f_{i}\left\|^{1 / p^{\prime}}\right\|_{\left(X_{\left[1 / p^{\prime}\right]}\right)^{\prime}},
\end{aligned}
$$

where $K^{\prime}=K\|\phi\|^{1 / p^{\prime}}$. Then $\phi$ satisfies the inequality in (i) of Theorem 2 for the homogeneous map $\phi_{p}$, i.e $\phi_{p}$ strongly dominates $\phi$.

Whenever $X(\mu)$ is an order continuous Banach function space with the Fatou property and $P: X(\mu) \rightarrow X(\mu)$ is a homogeneous bounded map strongly dominated by $\phi_{p}$, the factorization in Theorem 2 reads as follows: there is $g \in X_{\left[1 / p^{\prime}\right]}$ such that for every $f \in X$ there is a function $h_{f} \in B_{L^{\infty}(\mu)}$ depending only on $f /\|f\|_{X}$ such that $P(f)=g h_{f} \phi_{p}(f)$. In other words, $P$ factors through the homogeneous map $\hat{\phi}_{p}$ given by $\hat{\phi}_{p}(f)=h_{f} \phi_{p}(f)$ as

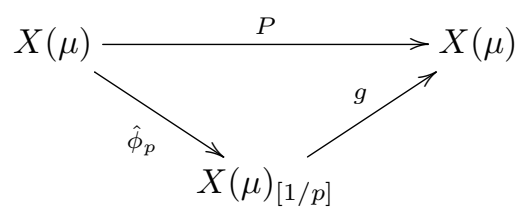

Moreover, by Proposition 2 the set $P\left(B_{X}\right)$ is uniformly $\mu$-absolutely continuous.

Let us see analogous results when considering bounded homogeneous maps with values on a general Banach space $E$. Note that, when $E=X(\mu)$ is order continuous and satisfies the Fatou property, condition (i) in the next result is weaker than strong domination of $S$ by $\phi_{p}$.

Let $X(\mu)$ be an order continuous Banach function space and let $E$ be a Banach space. The following statements for a bounded homogeneous operator $S: X(\mu) \rightarrow$ $E$ and a function $0<g \in X_{\left[1 / p^{\prime}\right]}$ are equivalent.

(i) For all functions $h \in X_{[1 / p]}$,

$$
\left\|S\left(h^{p}\right)\right\| \leq\left\|h^{p}\right\|_{X}^{1 / p^{\prime}} \cdot\|g h\|_{X} .
$$

(ii) The homogeneous operator $S$ factorizes through

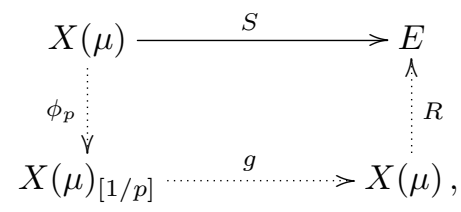


where $R$ is a bounded homogeneous operator with $\|R\| \leq 1$.

In this case, $S$ satisfies that $\lim _{\mu(A) \rightarrow 0} \sup _{f \in B_{X}}\left\|S\left(f \chi_{A}\right)\right\|=0$.

Proof. (i) $\Rightarrow$ (ii). Let us prove that the operator $S$ factors through the proposed scheme. The map $\phi_{p}(h)=h^{1 / p}\|h\|^{1 / p^{\prime}}, h \in X$, is a bijection, and

$$
\phi_{p}^{-1}(f)=\frac{f^{p}}{\left\|f^{p}\right\|_{X}^{1 / p^{\prime}}}, \quad f \in X .
$$

Clearly, since $g \in X_{\left[1 / p^{\prime}\right]}$ we have that the multiplication map $f \rightarrow g f$ is well defined and continuous. Define $R$ as follows. If $h \in g X_{[1 / p]}$, then

$$
R(h):=S\left(\phi_{p}^{-1}(h / g)\right)=S\left(\frac{(h / g)^{p}}{\left\|(h / g)^{p}\right\|_{X}^{1 / p^{\prime}}}\right),
$$

and $R(h)=0$ for the functions $h$ that do not belong to $g X_{[1 / p]}$. Note that the map is homogeneous.

Let us show that $R$ is bounded. Let $h \in g X_{[1 / p]}$. Then

$$
\begin{gathered}
\|R(h)\|=\left\|S\left(\phi_{p}^{-1}(h / g)\right)\right\|=\left\|S\left(\frac{(h / g)^{p}}{\left\|(h / g)^{p}\right\|_{X}^{1 / p^{\prime}}}\right)\right\| \\
=\frac{\left\|S\left((h / g)^{p}\right)\right\|}{\left\|(h / g)^{p}\right\|_{X}^{1 / p^{\prime}}} \leq \frac{\left\|(h / g)^{p}\right\|_{X}^{1 / p^{\prime}}\|h\|_{X}}{\left\|(h / g)^{p}\right\|_{X}^{1 / p^{\prime}}} \leq\|h\|_{X} .
\end{gathered}
$$

(ii) $\Rightarrow$ (i). Is the consequence of the following calculation. If $h \in X_{[1 / p]}$, then $h^{p} \in X$ and so

$$
\left\|S\left(h^{p}\right)\right\|=\left\|R\left(g \phi_{p}\left(h^{p}\right)\right)\right\| \leq\|R\|\left\|g\left(h^{p}\right)^{1 / p}\right\| h^{p}\left\|_{X}^{1 / p^{\prime}}\right\|_{X} \leq\|g h\|_{X}\left\|h^{p}\right\|_{X}^{1 / p^{\prime}} .
$$

The last assertion $\lim _{\mu(A) \rightarrow 0} \sup _{f \in B_{X}}\left\|S\left(f \chi_{A}\right)\right\|=0$ follows from the calculations below and the order continuity of $X_{\left[1 / p^{\prime}\right]}$. Let $f \in X$ then

$$
\begin{aligned}
\left\|S\left(f \chi_{A}\right)\right\| & \leq\left\|f \chi_{A}\right\|_{X}^{1 / p^{\prime}}\left\|g f^{1 / p} \chi_{A}\right\|_{X} \\
& \leq\left\|f \chi_{A}\right\|_{X}^{1 / p^{\prime}}\left\|g \chi_{A}\right\|_{X_{\left[1 / p^{\prime}\right]}}\left\|f^{1 / p} \chi_{A}\right\|_{X_{[1 / p]}} \\
& =\left\|f \chi_{A}\right\|_{X}^{1 / p^{\prime}}\left\|g \chi_{A}\right\|_{X_{\left[1 / p^{\prime}\right]}}\left\|f \chi_{A}\right\|_{X}^{1 / p} \\
& =\left\|f \chi_{A}\right\|_{X}\left\|g \chi_{A}\right\|_{X_{\left[1 / p^{\prime}\right]}} \leq\|f\|_{X}\left\|g \chi_{A}\right\|_{X_{\left[1 / p^{\prime}\right]}}
\end{aligned}
$$

Let us summarize in the next theorem some sufficient conditions for a homogeneous operator to satisfy that the ball of $X$ is uniformly $\mu$-absolutely continuous with respect to the seminorm $\|T(\cdot)\|$. We need the following lemma.

Let $E$ be a Banach space. The norm limit $T$ of a sequence $\left(S_{n}\right)_{n}$ of homogeneous bounded operators $S_{n}: X(\mu) \rightarrow E$ satisfying

$$
\lim _{\mu(A) \rightarrow 0} \sup _{f \in B_{X}}\left\|S_{n}\left(f \chi_{A}\right)\right\|=0
$$

for each $n$, is bounded, homogeneous and satisfies

$$
\lim _{\mu(A) \rightarrow 0} \sup _{f \in B_{X}}\left\|T\left(f \chi_{A}\right)\right\|=0 .
$$


Proof. Let $T$ be the norm limit of the sequence $\left(S_{n}\right)_{n}$. It is clearly homogeneous and bounded. Let $\varepsilon>0$ and take $n_{0}$ such that for all $n \geq n_{0},\left\|S_{n}-T\right\|<\varepsilon / 2$. Using the triangular inequality, we find that there is $\delta>0$ such that if $\mu(A)<\delta$,

$$
\sup _{f \in B_{X}}\left\|T\left(f \chi_{A}\right)\right\| \leq \varepsilon / 2+\sup _{f \in B_{X}}\left\|S_{n_{0}}\left(f \chi_{A}\right)\right\|<\varepsilon .
$$

Let $X(\mu)$ be an order continuous Banach function space and let $T: X(\mu) \rightarrow E$ be a bounded homogeneous operator. Each of the followings statements implies the next one.

(i) $X$ has the Fatou property and there is a sequence of solid homogeneous order bounded sets $\left(B_{n}\right)_{n}$ in $X(\mu)$ and a sequence of bounded homogeneous maps $R_{n}: X(\mu) \rightarrow E$ such that $R_{n} \circ \phi_{B_{n}}$ converges to $T$ in the norm.

(ii) $X(\mu)$ has the Fatou property and there is a sequence of solid homogeneous sets $B_{n} \subseteq X$ such that their characteristic homogeneous operators are strongly dominated by $\phi_{p}$

and a sequence of bounded homogeneous maps $R_{n}: X(\mu) \rightarrow E$ such that $R_{n} \circ \phi_{B_{n}} \rightarrow T$ in the operator norm.

(iii) $X(\mu)$ has the Fatou property and there is a sequence of homogeneous maps $P_{n}: X \rightarrow X$ that are strongly dominated by $\phi_{p}$ and a sequence of bounded homogeneous maps $R_{n}: X(\mu) \rightarrow E$ such that $R_{n} \circ P_{n}$ converges to $T$ in the norm.

(iv) $T$ is the norm limit of a sequence $S_{n}$ of homogeneous operators factoring as

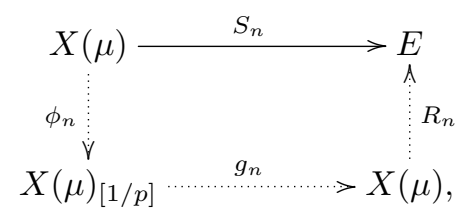

where $R_{n}$ are bounded homogeneous maps, $g_{n} \in X_{\left[1 / p^{\prime}\right]}$ and $\left|\phi_{n}\right| \leq\left|\phi_{p}\right|$.

(v) There is a sequence of homogeneous operators $S_{n}$ so that there are functions $g_{n} \in X_{\left[1 / p^{\prime}\right]}$ such that

$$
\left\|S_{n}(f)\right\| \leq\|f\|_{X}^{1 / p^{\prime}} \cdot\left\|g_{n} f^{1 / p}\right\|_{X}, \quad f \in X,
$$

and $S_{n} \rightarrow T$ in the operator norm.

(vi) The operator $T$ satisfies

$$
\lim _{\mu(A) \rightarrow 0} \sup _{f \in B_{X}}\left\|T\left(f \chi_{A}\right)\right\|=0
$$

Proof. (i) $\Rightarrow$ (ii) is a consequence of Proposition 2. (ii) clearly implies (iii). If $X(\mu)$ has the Fatou property, then (iii) is equivalent to (iv) by Proposition 2. The same computations that proves (ii) $\Rightarrow$ (i) in Proposition 2 gives that (iv) implies (v). Proposition 2 and Lemma 2 give (v) implies (vi). 


\section{COMPACT LiNEAR OPERATORS}

Compactness of operators between Banach spaces is nowadays well understood, and there are also a lot of characterizations of this property when the spaces involved are Banach function spaces. Let $X(\mu)$ be an order continuous Banach function space. It is clear that a homogeneous operator $T: X(\mu) \rightarrow E$ that factors through a uniformly $\mu$-absolutely continuous (non necessarily linear) homogeneous map and an essentially compact operator is compact (see [9, Proposition 3.7.4] or the argument in the proof of Proposition 3 below). In this section we are interested in analyzing how far the converse of this statement is true, that is, if every compact operator can be factored in this way.

Let $T: X(\mu) \rightarrow E$ be a compact operator. Then for each $\varepsilon>0$ we can consider the set $T\left(S_{X}\right)$, that is relatively compact in $E$. A simple compactness argument provides a number of functions $f_{1}, \ldots, f_{n} \in S_{X}$ such that the balls $B_{\varepsilon}\left(T\left(f_{i}\right)\right)$ of radius $\varepsilon$ and centers $T\left(f_{i}\right)$ cover $T\left(S_{X}\right)$. An inductive disjointification with the sets $T^{-1}\left(B_{\varepsilon}\left(T\left(f_{i}\right)\right)\right)$ provides a finite disjoint family $B_{1}, \ldots, B_{n}$ of subsets that covers $S_{X}$. Define the homogeneous map $P_{\varepsilon}(f):=\|f\| f_{i}$ if $f /\|f\| \in B_{i}, i=1, \ldots, n$. Note that $P_{\varepsilon}\left(B_{X}\right)$ is order bounded. For each $f \in X(\mu)$ there is $i \in\{1, \ldots, n\}$ such that $\frac{f}{\|f\|} \in B_{i}$. Then,

$$
\left\|T(f)-T\left(P_{\varepsilon}(f)\right)\right\|=\|f\|\left\|T\left(\frac{f}{\|f\|}\right)-T\left(f_{i}\right)\right\| \leq\|f\| \varepsilon .
$$

Hence,

$$
\lim _{\varepsilon \rightarrow 0}\left\|T-T \circ P_{\varepsilon}\right\|=\lim _{\varepsilon \rightarrow 0} \sup _{f \in B_{X}}\left\|T(f)-T\left(P_{\varepsilon}(f)\right)\right\|=0 .
$$

Therefore, $T$ is the norm limit of the sequence of homogeneous maps $\left(T \circ P_{1 / n}\right)_{n}$, where obviously each of them factors through $X$ by means of the order bounded homogeneous map $P_{1 / n}$. This construction proves in fact the following

Let $X(\mu)$ be an order continuous Banach function space. An operator $T$ : $X(\mu) \rightarrow E$ is compact if and only if it is essentially compact and there is an order continuous Banach function space $Z(\nu)$ containing $X$ such that $T$ extends to $Z$ and a sequence of uniformly $\nu$-absolutely continuous homogeneous maps $Q_{n}: X \rightarrow Z$ satisfying that $T$ is the norm limit of the sequence $\left(T \circ Q_{n}\right)_{n}$.

Proof. The comments above prove the factorization for a compact operator, with $Z=X$. For the converse, just note that for each $n$ the set $Q_{n}\left(B_{X}\right)$ is uniformly $\nu$-absolutely continuous and essentially compact operators map this kind of sets to relatively compact ones ([12, Proposition 2.41]). Therefore, the norm convergence of $\left(T \circ Q_{n}\right)_{n}$ to $T$ allows to approximate $T\left(B_{X}\right)$ by relatively compact sets, so it is compact.

The next result allows to characterize when a particular sequence of homogeneous maps $\left(\phi_{n}\right)_{n}$ taking values in a Banach function space gives for an operator $T$ a uniformly $\nu$-absolutely continuous homogeneous map $\phi$ such that $T=T \circ \phi$. We will see that not all sequences of homogeneous operators provide such a good factorization, although after Proposition 3 we know that it is always possible to approximate a compact operator by means of homogeneous maps that are uniformly $\nu$-absolutely continuous. Recall that we always have that $X \subseteq L^{1}\left(m_{T}\right)$ for a $\mu$ determined operator $T: X \rightarrow E$, and $T$ can be extended to any order continuous Banach function space $Z(\eta)$ such that $X \subseteq Z \subseteq L^{1}\left(m_{T}\right)$. 
Let $X(\mu)$ be an order continuous Banach function space. The following assertions for a compact $\mu$-determined operator $T: X(\mu) \rightarrow E$ and a Rybakov measure $\nu$ for $m_{T}$ are equivalent.

(i) There is an order continuous Banach function space $Z(\eta), X \subseteq Z \subseteq$ $L^{1}\left(m_{T}\right)$, and a sequence of uniformly $\eta$-absolutely continuous homogeneous maps $\left(\phi_{n}\right)_{n}$ from $X$ to $Z$ such that $T=\lim _{n} T \circ \phi_{n}$ and

$$
\lim _{n, m} \sup _{f \in B_{X}} \sup _{A \in \Sigma}\left\|T\left(\left(\phi_{n}(f)-\phi_{m}(f)\right) \chi_{A}\right)\right\|=0 .
$$

(ii) There is a uniformly $\nu$-absolutely continuous homogeneous map $\phi: X \rightarrow$ $L^{1}\left(m_{T}\right)$ such that $T=T \circ \phi$.

Proof. (ii) $\Rightarrow$ (i). Take $\phi_{n}=\phi$ and $\eta=\nu$. (i) $\Rightarrow$ (ii). Since $\phi_{n}: X \rightarrow Z(\eta) \subseteq L^{1}\left(m_{T}\right)$, $\nu$ is a Rybakov measure for $m_{T}$ and the following inequalities hold

$$
\lim _{n, m} \sup _{f \in B_{X}}\left\|\phi_{n}(f)-\phi_{m}(f)\right\|_{L^{1}\left(m_{T}\right)} \leq 2 \lim _{n, m} \sup _{f \in B_{X}} \sup _{A \in \Sigma}\left\|T\left(\left(\phi_{n}(f)-\phi_{m}(f)\right) \chi_{A}\right)\right\|,
$$

we have that the $\operatorname{limit} \phi(f):=\lim _{n} \phi_{n}(f)$ is well defined for every $f \in X$ and belongs to $L^{1}\left(m_{T}\right)$, and $\left(\phi_{n}\right)_{n}$ norm converges to $\phi$, that is also a homogeneous map. Let us show that it is uniformly $\nu$-absolutely continuous. Let $\varepsilon>0$ and fix $n_{0} \in \mathbb{N}$ such that $\left\|\phi-\phi_{n_{0}}\right\|<\varepsilon$. By the fact that $\phi_{n_{0}}$ is uniformly $\eta$-absolutely continuous, there is $\rho>0$ such that $\eta(A)<\rho$ implies $\left\|\phi_{n_{0}}(f) \chi_{A}\right\|_{L^{1}\left(m_{T}\right)} \leq\left\|\phi_{n_{0}}(f) \chi_{A}\right\|_{Z}<\varepsilon$ for all $f \in B_{X}$. Now, since $T$ is $\mu$-determined we have that $\mu \ll \nu$, and so $\mu \approx \eta \approx \nu$ by $X \subseteq Z \subseteq L^{1}\left(m_{T}\right)$. Thus there is $\delta>0$ such that $\nu(A)<\delta$ implies $\eta(A)<\rho$. Then there is $n_{0}$ such that

$$
\left\|\phi(f) \chi_{A}\right\|_{L^{1}\left(m_{T}\right)} \leq\left\|\left(\phi(f)-\phi_{n_{0}}(f)\right) \chi_{A}\right\|_{L^{1}\left(m_{T}\right)}+\left\|\phi_{n_{0}}(f) \chi_{A}\right\|_{L^{1}\left(m_{T}\right)}<2 \varepsilon
$$

for all $A \in \Sigma$ such that $\nu(A)<\delta$. Therefore, $\phi$ is also uniformly $\nu$-absolutely continuous. This gives the result.

Let us show an example of a compact operator satisfying the statements in Proposition 3 .

(1) Let $X$ be a reflexive Banach function space over $\mu$. Take a $\mu$-determined compact operator $T: L^{1}(\mu) \rightarrow E$, and consider its restriction $T_{X}$ to $X$. Since $L^{1}\left(m_{T}\right)=L^{1}\left(\left|m_{T}\right|\right)$ we have that $B_{X}$ is a relatively weakly compact subset of $L^{1}\left(\left|m_{T}\right|\right)$ and so it is approximately order bounded (uniformly $\left|m_{T}\right|$-absolutely continuous). So in this case the inclusion map $i: X \rightarrow L^{1}\left(m_{T}\right)$ provides a suitable homogeneous map $\phi=i$ such that $T \circ \phi=T \circ i=T_{X}$. However, the definition of $\phi$ as $i$ does not provide always the desired factorization, as will be shown in Example 3 .

(2) Consider the integration map $I: L^{1}[0,1] \rightarrow \mathbb{R}$ defined by Lebesgue measure. For each $n \in \mathbb{N}$ we define $\phi_{n}(f):=k / n\|f\| \chi_{[0,1]}$ if $\int f /\|f\| d x \in\left(\frac{k-1}{n}, \frac{k}{n}\right]$ for $-n+1 \leq k \leq n$, and $\phi_{n}(f):=-1\|f\| \chi_{[0,1]}$ if $\int f /\|f\| d x=-1$. Clearly $\lim _{n} I \circ \phi_{n}=$ I. In fact, if we define $\phi(f):=\left(\int f d x\right) \chi_{[0,1]}, f \in L^{1}[0,1]$, we have that $\lim _{n} \phi_{n}=\phi$ in the norm.

Clearly, each essentially compact operator $T$ that satisfies the requirements of the result above is compact. However, there are sequences of homogeneous maps approximating compact operators as above that do not converge to a homogeneous map in any Banach function space. Let us show this with an example.

Let $F$ be a Banach lattice. Consider a compact integration map $I_{m}: L^{1}(m) \rightarrow$ $F$ associated to a positive vector measure $m$. Then it is known that $L^{1}(m)=$ 
$L^{1}(|m|)$. Consider a sequence of homogeneous maps $\left(\phi_{n}\right)_{n}$ from $L^{1}(m)$ to $L^{1}(m)$ approximating $I_{m}$ in the sense given in Proposition 3 that satisfies $\phi_{n}\left(f \chi_{A}\right) \chi_{A}=$ $\phi_{n}\left(f \chi_{A}\right)$ for all $n$ and $A \in \Sigma$. Suppose that it converges in $L^{1}(m)$ to a homogeneous map $\phi$ as in Proposition 3. Then $\phi$ satisfies also the equality

$$
\phi\left(f \chi_{A}\right) \chi_{A}=\phi\left(f \chi_{A}\right)
$$

for $f \in L^{1}(m)$ and $A \in \Sigma$. Thus, the set $\phi\left(B_{L^{1}(m)}\right)$ is uniformly $\nu$-absolutely continuous, where $\nu$ is a Rybakov measure for $m$. Since $\nu$ and $m$ are equivalent measures, they are also equivalent to $|m|$, and so the set $\phi\left(B_{L^{1}(m)}\right)$ is uniformly $|m|$-absolutely continuous. The solid hull $C$ of $\left|\phi\left(B_{L^{1}(m)}\right)\right|$ is still uniformly $\nu$ absolutely continuous. This implies that it is relatively weakly compact, by the characterization of weakly compact sets in $L^{1}$-spaces. Since in this case the space $L^{1}(m)$ is not reflexive, we can find a function $f \in B_{L^{1}(m)}$ not belonging to $C$. So there is a non-null measurable set $B \in \Sigma$ such that $\left|f \chi_{B}\right|>g \chi_{B}$ for all $g \in C$ : otherwise we should have that $f \in C$, since $C$ is solid. Therefore the positivity of $m$ gives

$$
\left.0<\left\||f| \chi_{B}-\phi\left(|f| \chi_{B}\right) \chi_{B}\right\|_{L^{1}(m)}=\| I_{m}\left(|f| \chi_{B}-\phi\left(|f| \chi_{B}\right)\right)\right) \|,
$$

which gives a contradiction. The required condition (1) on each $\phi_{n}$ is satisfied for example for $\phi_{n}(f)=\operatorname{sign}(f)\|f\|\left(|f| /\|f\| \wedge n \chi_{\Omega}\right)$. Also homogeneous maps that are strongly dominated by $\phi_{p}$ satisfy this requirement.

It is well-known that compactness and essential compactness are not equivalent properties for operators on Banach function spaces. An example that will be useful also in the next section is the Volterra operator. Let $1<r<\infty$. Consider the Volterra operator $V_{r}: L^{r}[0,1] \rightarrow L^{r}[0,1]$ given by $V_{r}(f)(x):=\int_{0}^{x} f(t) d t, x \in[0,1]$, $f \in L^{r}[0,1]$. It is known to be compact (see Example 3.10 in [12]). However, the integration map $I_{\nu_{r}}: L^{1}\left(\nu_{r}\right) \rightarrow L^{r}[0,1]$ associated to the Volterra measure $\nu_{r}(A):=V_{r}\left(\chi_{A}\right)$ for any Borel set $A$ in $[0,1]$, is not compact, while it is essentially compact as a consequence of $V_{r}$ being compact (see Remark 3.57(ii) and Proposition 3.52 in [12]). The first aim of this section is to characterize the difference between these two properties for general operators from order continuous Banach function spaces into locally convex spaces.

Proposition 3 suggests that uniformly $\mu$-absolutely continuous maps are the natural elements appearing in a reasonable factorization for compact operators. A relevant class of $\mu$-absolutely continuous sets is the one defined by subsets of the unit ball of the $p$-convexifications $X_{[1 / p]}$ of $X(\mu)$ when considered as subsets of $X(\mu)$. As far as we know no specific description of compactness using the $p$-convexifications of the spaces - their $1 / p$-th powers in the terminology of [12] - has been given. We will show how useful are $p$-convexifications for our purposes. Actually, our second aim of this section is to characterize compact operators as limits of homogeneous maps that factor though a space $X_{[1 / p]}$ for a given $1<p<\infty$.

We need the next lemma, that is a consequence of Hölder's inequality $\|g h\|_{X} \leq$ $\|g\|_{X_{[1 / p]}}\|h\|_{X_{\left[1 / p^{\prime}\right]}}$ for $g \in X_{[1 / p]}$ and $h \in X_{\left[1 / p^{\prime}\right]}$ (see [6, Lemma 3.8] and Proposition 2.41 in [12] for similar arguments).

Let $1<p<\infty$. Let $X(\mu)$ be an order continuous Banach function space and $E$ be a Banach space. A continuous operator $T: X(\mu) \rightarrow E$ is essentially compact if and only if for every $h \in X_{\left[1 / p^{\prime}\right]}$ the map $T_{h}: X_{[1 / p]} \rightarrow E$ given by $T_{h}(\cdot):=T(h \cdot)$, is compact. 
Proof. Assume that $T$ is essentially compact. Then $\left\{T\left(\chi_{B \cap A}\right): A \in \Sigma\right\}$ is relatively compact for all $B \in \Sigma$, and so the operator $T_{h}$ is compact for simple functions $h$. Let now $h$ be an arbitrary function in $X_{\left[1 / p^{\prime}\right]}$. Since the set of simple functions is dense in $X_{\left[1 / p^{\prime}\right]}$, for $\varepsilon>0$ there is a simple function $h_{0}$ such that a $\left\|h-h_{0}\right\|_{X_{\left[1 / p^{\prime}\right]}}<\varepsilon$. Then, if $g \in X_{[1 / p]}$,

$$
\begin{aligned}
\left\|T_{h}(g)-T_{h_{0}}(g)\right\| & \leq\left\|T\left(\left(h-h_{0}\right) g\right)\right\| \\
& \leq\|T\|\left\|h-h_{0}\right\|_{X_{\left[1 / p^{\prime}\right]}}\|g\|_{X_{[1 / p]}}<\varepsilon\|T\|\|g\|_{X_{[1 / p]}} .
\end{aligned}
$$

Thus, the fact that compact operators are closed for the operator norm gives that $T_{h}$ is compact for all $h \in X_{\left[1 / p^{\prime}\right]}$. The converse is obvious.

Let $1 \leq p<\infty$. Let $X(\mu)$ be an order continuous Banach function space and let $E$ be a Banach space. The following statements for a continuous linear operator $T: X \rightarrow E$ are equivalent:

(i) $T$ is compact.

(ii) $T$ is essentially compact and for every $\varepsilon>0$ there exists $g_{\varepsilon} \in X_{\left[1 / p^{\prime}\right]}$ such that $T\left(B_{X}\right) \subset T\left(g_{\varepsilon} B_{X_{[1 / p]}}\right)+\varepsilon B_{E}$.

(iii) $T$ is essentially compact and for every $\varepsilon>0$ there exists $K_{\varepsilon}>0$ such that $T\left(B_{X}\right) \subset T\left(K_{\varepsilon} B_{X_{[1 / p]}}\right)+\varepsilon B_{E}$.

Proof. (i) $\Rightarrow$ (ii). By the compactness argument described before Proposition 3, given $\epsilon>0$ there exist finitely many $f_{1}, \ldots, f_{l} \in B_{X}$ such that

$$
T\left(B_{X}\right) \subset \cup_{i=1}^{l} T\left(f_{i}\right)+\epsilon B_{E} .
$$

For each $i=1, \ldots, l$ write $f_{i}=g_{i} h_{i}$, where $g_{i} \in B_{X_{\left[1 / p^{\prime}\right]}}$ and $h_{i} \in B_{X_{[1 / p]}}$. Notice that by the lattice properties of the norm of $X_{[1 / p]}$, for any $\hat{g}_{1}, \hat{g}_{2} \in X_{\left[1 / p^{\prime}\right]}$ satisfying $\left|\hat{g}_{1}\right| \leq\left|\hat{g}_{2}\right|$ a.e., we have that $\hat{g}_{1} B_{X_{[1 / p]}} \subseteq\left|\hat{g}_{2}\right| B_{X_{[1 / p]}}$. Indeed, given $h \in B_{X_{[1 / p]}}$ define $g(x):=\frac{\hat{g}_{1}(x)}{\left|\hat{g}_{2}(x)\right|}$ if $\hat{g}_{2}(x) \neq 0$ and $g(x)=0$ if $\hat{g}_{2}(x)=0$. Then $\hat{g}_{1}=g\left|\hat{g}_{2}\right|$ and $|h g|^{p} \leq|h|^{p}$. By the ideal property of Banach function spaces it follows that $h g \in B_{X_{[1 / p]}}$. Therefore $\hat{g}_{1} h=g\left|\hat{g}_{2}\right| h \in\left|\hat{g}_{2}\right| B_{X_{[1 / p]}}$.

Define $g_{\epsilon}=\sum_{i=1}^{l}\left|g_{i}\right|$. Since $\left|g_{i}\right| \leq g_{\epsilon}$, we obtain that $g_{i} B_{X_{[1 / p]}} \subset g_{\epsilon} B_{X_{[1 / p]}}$, and so $T\left(g_{i} B_{X_{[1 / p]}}\right) \subset T\left(g_{\epsilon} B_{X_{[1 / p]}}\right)$ for all $i=1, \ldots, l$. Therefore,

$$
\cup_{i=1}^{l} T\left(g_{i} B_{X_{[1 / p]}}\right)+\epsilon B_{E} \subset T\left(g_{\epsilon} B_{X_{[1 / p]}}\right)+\epsilon B_{E}
$$

and (ii) is proved.

Let us prove (ii) $\Rightarrow$ (iii). Let $\varepsilon>0$ and consider $g_{\frac{\varepsilon}{2}} \in X_{\left[1 / p^{\prime}\right]}$ given by (ii). By continuity there exists $\delta>0$ such that $T\left(\delta B_{X}\right) \subset \frac{\varepsilon}{2} B_{E}$. Since simple functions are dense in $X_{\left[1 / p^{\prime}\right]}$, we find a simple function $s$ such that $\left\|\left(g_{\frac{\varepsilon}{2}}-s\right) f\right\|_{X} \leq \| g_{\frac{\varepsilon}{2}}-$ $s \|_{X_{\left[1 / p^{\prime}\right]}}<\delta$ for every $f \in B_{X_{[1 / p]}}$, and so $T\left(\left(g_{\frac{\varepsilon}{2}}-s\right) B_{X_{[1 / p]}}\right) \subseteq \frac{\varepsilon}{2} B_{E}$. Take $K_{\varepsilon}:=\|s\|_{L^{\infty}(\mu)}$. Then, again by the ideal property of $X_{[1 / p]}$, we obtain

$$
\begin{gathered}
T\left(B_{X}\right) \subseteq T\left(g_{\frac{\varepsilon}{2}} B_{X_{[1 / p]}}\right)+\frac{\varepsilon}{2} B_{E} \subseteq T\left(s B_{X_{[1 / p]}}\right)+T\left(\left(g_{\frac{\varepsilon}{2}}-s\right) B_{X_{[1 / p]}}\right)+\frac{\varepsilon}{2} B_{E} \\
\subseteq T\left(s B_{X_{[1 / p]}}\right)+\frac{\varepsilon}{2} B_{E}+\frac{\varepsilon}{2} B_{E} \subseteq T\left(K_{\varepsilon} B_{X_{[1 / p]}}\right)+\varepsilon B_{E} .
\end{gathered}
$$

This gives the result.

We prove (ii) $\Rightarrow$ (i) (the proof for (iii) $\Rightarrow$ (i) is the same). Take an arbitrary $\varepsilon>0$ and chose $g_{\varepsilon} \in X_{\left[1 / p^{\prime}\right]}$ such that $T\left(B_{X}\right) \subset T\left(g_{\varepsilon} B_{X_{[1 / p]}}\right)+\varepsilon B_{E}$. By Lemma 3 the set $T\left(g_{\varepsilon} B_{X_{[1 / p]}}\right)$ is relatively compact in $E$. Then we have shown that for 
any $\varepsilon>0$, there is a relatively compact set $T\left(g_{\varepsilon} B_{X_{[1 / p]}}\right)$ such that $T\left(B_{X}\right) \subset$ $T\left(g_{\varepsilon} B_{X_{[1 / p]}}\right)+\varepsilon B_{E}$. By Grothendieck's characterization of compactness it follows that $T\left(B_{X}\right)$ is relatively compact.

Notice that in fact the requirement of order continuity is just needed to assure that simple functions are dense in the involved spaces, so the result is also true just assuming this fact. This let us include the relevant case of $L^{\infty}(\mu)$ which can be considered in a sense as a limit case of the above theorem in which the second assumption in (ii) and (iii) is trivially satisfied, although the space is not order continuous (see Proposition 2.41 in [12]). In this case, we have that $X_{[1 / p]}=$ $L^{\infty}(\mu)_{[1 / p]}=L^{\infty}(\mu)=X$ isometrically, and so an operator $T: L^{\infty}(\mu) \rightarrow E$ is compact if and only if its essential range is relatively compact, since $T\left(B_{L^{\infty}(\mu)}\right)$ is included in the closure of the absolutely convex hull of the essential range of $T$.

Next result, that is already known (see the arguments that prove Corollaries 5.16 and 5.20 of [12]) is a direct consequence of Lemma 3.

Let $1<p<\infty$. Let $X(\mu)$ be an order continuous Banach function space and let $E$ be a Banach space. If $T: X(\mu) \rightarrow E$ is a continuous essentially compact operator then the restriction $\left.T\right|_{X_{[1 / p]}}: X_{[1 / p]} \rightarrow E$ is compact.

1.- Both conditions in (ii) and (iii) are necessary for the result above to be true, even if the operator $T$ involved is positive. Let us see some examples.

(i) Take $X(\mu)=L^{1}[0,1]$ and $1<p<\infty$. Then $X_{[1 / p]}=L^{p}[0,1]$ isometrically. Consider a partition $\left\{A_{i}\right\}_{i=1}^{\infty}$ of $[0,1]$, where $\mu\left(A_{i}\right)>0$ for all $i \in \mathbb{N}$. Take a sequence of non-null measurable sets $\left\{B_{i}\right\}_{i=1}^{\infty}$ such that $B_{i} \subseteq A_{i}$ for all $i$ and $r_{i}:=\mu\left(B_{i}\right) / \mu\left(A_{i}\right) \downarrow 0$ and the operator $T: L^{1}[0,1] \rightarrow \ell^{1}$ given by $T(f):=$ $\sum_{i=1}^{\infty}\left(\int_{B_{i}} f d \mu\right) e_{i} \in \ell^{1}, f \in L^{1}[0,1]$. Let us show that the requirement on $\left\{T\left(\chi_{A}\right)\right.$ : $A \in \Sigma\}$ of being relatively compact is fulfilled. Consider the sequence $\left\{r_{i} e_{i}\right\}_{i=1}^{\infty}$. For every $A \in \Sigma$,

$$
T\left(\chi_{A}\right)=\sum_{i=1}^{\infty} \mu\left(B_{i} \cap A\right) e_{i} \leq \sum_{i=1}^{\infty} \mu\left(A_{i}\right)\left(r_{i} e_{i}\right)
$$

in the order of $\ell^{1}$, and so each $T\left(\chi_{A}\right)$ is in the closure of the convex hull of $\left\{r_{i} e_{i}\right\}_{i=1}^{\infty}$. Thus, it is relatively compact. In fact, any $\ell^{1}$-valued continuous operator defined on an order continuous Banach function space is essentially compact (see [12, Lemma $3.53(\mathrm{v})]$ ). However, since for every $i$ we can find a positive norm one function $f_{i}$ of $L^{1}(\mu)$ with support in $B_{i}$, the set $T\left(B_{L^{1}[0,1]}\right)$ includes all $\left\{e_{i}\right\}_{i=1}^{\infty}$, and so $T$ is not compact. Consequently, there is $\varepsilon>0$ such that there is no $K$ such that $T\left(B_{L^{1}[0,1]}\right) \subseteq K T\left(B_{L^{p}[0,1]}\right)+\varepsilon B_{\ell^{1}}$.

(ii) Although operators of compact range with domain in an $L^{1}$-space allow sometimes good characterizations - due mainly to 1-concavity of $L^{1}$-, this fact is not crucial in the example above. Consider $p \geq q \geq 1$ and the class of spaces $E_{p, q}=\left(\oplus_{p}\right)_{i=1}^{\infty} L^{q}\left(A_{i},\left.\mu\right|_{A_{i}}\right)$, where the $A_{i}^{\prime} s$ are chosen as in (i), with the norm

$$
\|\| f \|_{p, q}:=\left(\sum_{i=1}^{\infty}\left\|\left.f\right|_{A_{i}}\right\|_{L^{q}[0,1]}^{p}\right)^{1 / p}, \quad f \in E_{p, q} .
$$

It is a Banach function space of Lebesgue measurable functions on $[0,1]$ over a measure $\mu_{a}$ given by $\mu_{a}(A):=\sum_{i=1}^{\infty} a_{i} \mu\left(A_{i} \cap A\right)$, where $a=\left(a_{i}\right)$ is any strictly positive element of $B_{\ell^{p^{\prime}}}$. Notice also that it contains $L^{1}[0,1]$. The same calculations that 
in the above case prove that $T$ defined as in $(\mathrm{i})-$ i.e. $T(\cdot):=\sum_{i=1}^{\infty}\left(\int_{B_{i}}(\cdot) d \mu\right) e_{i}$ is continuous also if defined from $E_{p, 1}$ to $\ell^{p}$, and its essential range $T$ is relatively compact, but the operator is not compact. Then, since $\left(E_{p, 1}\right)_{[1 / p]}=E_{p^{2}, p}$, we get that there is an $\varepsilon>0$ such that $T\left(B_{E_{p, 1}}\right) \nsubseteq K T\left(B_{E_{p^{2}, p}}\right)+\varepsilon B_{\ell^{p}}$ for all constants $K>0$.

More examples of $T$ having compact essential range and not being compact can be found in Example 3.49 of [12] (see also the comments after Proposition 3.48 in $[12])$.

2.- The extreme case of operators from $L^{\infty}$ can be considered also as a particular instance of our result. In the case of considering the operator $T_{\infty}$ as the restriction of the operator $T$ in 1.(i) to $L^{\infty}[0,1]$, Remark 3 applies: $T$ is compact, since its essential range is compact and $B_{L^{\infty}[0,1]}=B_{L^{\infty}[0,1]_{[1 / p]}}$.

Let us show a sufficient condition for $T$ to be compact by choosing a particular element $g \in B_{X_{[1 / p]}}$ for each $f \in X$ and $K$ in the criterion given by Theorem 3 . Our aim is to show the role of the corresponding $p$-convexifications.

Let $1<p<\infty$. Let $X(\mu)$ be an order continuous Banach function space, $E$ be a Banach space, and $T: X(\mu) \rightarrow E$ be an essentially compact continuous linear operator. Let $\Phi: B_{X} \rightarrow B_{X_{[1 / p]}}$ be a function. If

$$
\lim _{K \rightarrow \infty} \sup _{f \in B_{X}}\left\|T\left(f \chi_{\{|f| \geq K|\Phi(f)|\}}\right)\right\|=0
$$

then $T$ is compact.

Proof. Let $1<p<\infty$ and $\varepsilon>0$. Choose $K_{\varepsilon} \geq 1$ such that

$$
\sup _{f \in B_{X}}\left\|T\left(f \chi_{\left\{|f| \geq K_{\varepsilon}|\Phi(f)|\right\}}\right)\right\|<\varepsilon .
$$

Let $f \in B_{X}$. Then for every $h \in B_{L^{\infty}(\mu)}$,

$$
\inf _{g \in B_{X_{[1 / p]}}}\left\|T\left(f-K_{\varepsilon} g\right)\right\| \leq\left\|T\left(f-K_{\varepsilon} \Phi(f) h\right)\right\| .
$$

Take now $h:=\frac{f}{K_{\varepsilon} \Phi(f)} \chi_{\left\{|f|<K_{\varepsilon}|\Phi(f)|\right\}}$, and notice that $h \in B_{L^{\infty}}$. Then

$$
\begin{gathered}
\inf _{g \in B_{X_{[1 / p]}}}\left\|T\left(f-K_{\varepsilon} g\right)\right\| \leq \| T\left(f-K_{\varepsilon} \Phi(f) \frac{f}{K_{\varepsilon} \Phi(f)} \chi_{\left.\left\{|f|<K_{\varepsilon}|\Phi(f)|\right\} \mid\right) \mid \|}\right. \\
=\left\|T\left(f \chi_{\left\{|f| \geq K_{\varepsilon}|\Phi(f)|\right\}}\right)\right\|<\varepsilon .
\end{gathered}
$$

Theorem 3 gives the result.

An example of application of this criterion - by putting $\Phi(f):=\chi_{\Omega}$ for all $f \in B_{X}$ and assuming that $\left\|\chi_{\Omega}\right\|=1-$ is the following well-known sufficient condition for compactness. If an essentially compact operator $T: X \rightarrow E$ satisfies

$$
\lim _{K \rightarrow \infty} \sup _{f \in B_{X}}\left\|T\left(f \chi_{\{|f| \geq K\}}\right)\right\|=0
$$

then it is compact. For the particular case of kernel operators, this kind of computation provides more specific sufficient conditions for compactness. Consider $\Omega=[0,1]$ and $\mu$ Lebesgue measure on $[0,1]$. Let $k:[0,1] \times[0,1] \rightarrow[0, \infty)$ be a kernel function, that is a Borel measurable function such that, for all $y \in[0,1]$ the 
function $k_{y}(x):=k(y, x), x \in[0,1]$, is $\mu$-integrable. Let $T_{k}$ be the associated kernel operator given by

$$
\left(T_{k} f\right)(y):=\int_{[0,1]} f(x) k(y, x) d x
$$

for any measurable function $f:[0,1] \rightarrow \mathbb{R}$ for which the above integral is defined for $y \in[0,1]$ a.e. Let us give an example. Assume that $1<p<\infty$ and let $T_{k}$ be an essentially compact kernel operator $T_{k}: L^{p}[0,1] \rightarrow Y(\nu)$ that admits an extension to $L^{1}[0,1]$. Although we know by Corollary 3 that $T_{k}$ is compact we are able to prove the compactness of $T_{k}$ as an application of Corollary 3. For a nonzero function $f \in B_{L^{p}[0,1]}$ and $K>0$, by Hölder's inequality we have

$$
\mu(\{|f|>K\}) \leq\left(\int_{[0,1]}|f|^{p} d \mu\right)^{1 / p}\left(\int_{[0,1]} \frac{1}{K^{p^{\prime}}} d \mu\right)^{1 / p^{\prime}} \leq \frac{1}{K} .
$$

A direct computation shows

$$
\left\|T_{k}\left(|f| \chi_{\{|f|>K\}}\right)\right\|_{Y} \leq\left\|T_{k}\right\| \frac{1}{K^{1 / p^{\prime}}}
$$

that tends to 0 uniformly on $f \in B_{L^{p}(\mu)}$ when $K \rightarrow \infty$. Corollary 3 gives now the compactness of $T_{k}$. This argument can be extended in the following way.

Let $T$ be an essentially compact (positive) kernel operator $T: X(\mu) \rightarrow Y(\nu)$ such that the kernel $k$ satisfies that

$$
\lim _{\mu(A) \rightarrow 0}\|\| \chi_{A} k(x, y)\left\|_{X^{\prime}}(y)\right\|_{Y}=0 .
$$

Then $T$ is compact.

Proof. Let $\epsilon>0$ and take $\delta>0$ such that

$$
\|\| \chi_{A} k(x, y)\left\|_{X^{\prime}}(y)\right\|_{Y}<\epsilon
$$

for all measurable sets $A$ with $\mu(A)<\delta$. If $0 \neq f \in B_{X(\mu)}, K>0$ and $X^{\prime}$ is the Köthe dual of $X$ then

$$
\begin{gathered}
\mu(\{|f|>K\})=\int_{\{|f|>K\}} d \mu=\int_{\{|f|>K\}}|f| \cdot \frac{1}{|f|} d \mu \\
\leq\|f\|_{X}\left\|\chi_{\{|f|>K\}} \frac{1}{|f|}\right\|_{X^{\prime}} \leq \frac{1}{K}\left\|\chi_{\Omega}\right\|_{X^{\prime}}<\delta,
\end{gathered}
$$

for $K$ large enough (not depending on $f$ ). Then,

$$
\begin{gathered}
\left\|\left(\int|f| \chi_{\{|f|>K\}}|k(x, y)| d x\right)(y)\right\|_{Y} \\
\leq\|\||f| \chi_{\{|f|>K\}}\left\|_{X} \cdot\right\| \chi_{\{|f|>K\}}|k(x, y)|\left\|_{X^{\prime}}(y)\right\|_{Y} \\
\leq\|\| \chi_{\{|f|>K\}}|k(x, y)|\left\|_{X^{\prime}}(y)\right\|_{Y}<\epsilon
\end{gathered}
$$

for all $f \in B_{X(\mu)}$. This proves that

$$
\sup _{f \in B_{X}}\left\|\left(\int f \chi_{\{|f|>K\}} k(x, y) d x\right)(y)\right\|_{Y} \rightarrow 0
$$

when $K \rightarrow \infty$. Corollary 3 (for $\Phi(\cdot)=\chi_{\Omega}$ ) gives the result. 
The sufficient condition in Corollary 3 can be relaxed when considering the optimal extension of the operator $T: X(\mu) \rightarrow E$, but then $\mu$-determination of $T$ is required. Recall that a linear operator $T: X(\mu) \rightarrow E$ is $\mu$-determined if each $m_{T}$-null set is $\mu$-null.

Let $X(\mu)$ be an order continuous Banach function space, $E$ be a Banach space and $T: X \rightarrow E$ be a $\mu$-determined operator. If $T$ is essentially compact and satisfies

$$
\lim _{\mu(A) \rightarrow 0} \sup _{f \in B_{X}}\left\|T\left(f \chi_{A}\right)\right\|=0
$$

then $T$ is compact.

Proof. Let us show a proof of this result using the vector measure $m_{T}$. We extend the operator $T$ to the space $L^{1}\left(m_{T}\right)$ by means of the integration map $I_{m_{T}}$ using the well known fact that $I_{m_{T}} \circ[i]=T$, where $[i]: X \rightarrow L^{1}\left(m_{T}\right)$ is the canonical identification of the elements of $X$ as $m_{T}$-integrable functions. Since $T$ is $\mu$-determined, $[i]$ is injective. Since $T$ is essentially compact, so is $I_{m_{T}}$. Note now that for $A \in \Sigma$,

$$
\sup _{f \in B_{X}}\left(\sup _{B \subseteq A}\left\|\int_{B} f \chi_{A} d m_{T}\right\|\right)=\sup _{f \in B_{X}} \sup _{B \subseteq A}\left\|T\left(f \chi_{B}\right)\right\|=\sup _{f \in B_{X}}\left\|T\left(f \chi_{A}\right)\right\| .
$$

So we get by using the equivalent expression for the norm of $L^{1}\left(m_{T}\right)$ given in the introduction that $[i](X)$ is a uniformly $\nu$-absolutely continuous subset of $L^{1}\left(m_{T}\right)$ for a Rybakov measure $\nu$ for $m_{T}$, since $\mu$ is absolutely continuous with respect to $\nu$. This is a consequence of the fact that $m_{T}$ is equivalent to $\mu$, and so $\nu$ is equivalent to $\mu$. Consequently, $T\left(B_{X}\right)=I_{m_{T}}\left([i]\left(B_{X}\right)\right)$ is a relatively compact subset of $E$.

Now we are in conditions of using the results on factorization of homogeneous maps of the previous section to analyze compact operators.

For a $\mu$-determined operator, each one of the assertions in Theorem 2 implies that $T$ is compact.

Let us refine now Theorem 3 in order to provide a characterization of compactness of a linear operator $T$ in terms of approximation of $T$ by means of homogeneous operators strongly dominated by $\phi_{p}$. Given a homogeneous subset $B \subset X(\mu)$, the characteristic homogenous operator $\phi_{B}$ is defined by $\phi_{B}(f):=f$ if $f \in B$ and $\phi_{B}(f):=0$ if $f \notin B$.

Let $E$ be a Banach space, $X(\mu)$ be an order continuous Banach function space with the Fatou property and $1<p<\infty$. The following statements for a linear operator $T: X(\mu) \rightarrow E$ are equivalent.

(i) $T$ is compact.

(ii) $T$ is essentially compact and there are $g_{n} \in X_{\left[1 / p^{\prime}\right]}$ and bounded homogeneous maps $\varphi_{n}: X \rightarrow X_{[1 / p]}$ such that $\varphi_{n}\left(B_{X}\right)$ is order bounded and the sequence $T \circ\left(g_{n} \cdot \varphi_{n}\right)$ converges to $T$ in the norm.

(iii) $T$ is essentially compact and there are bounded homogeneous maps $\varphi_{n}$ : $X \rightarrow X$ such that $\varphi_{n}\left(B_{X}\right)$ is order bounded and the sequence $T \circ \varphi_{n}$ converges to $T$ in the norm.

(iv) $T$ is essentially compact and there are order bounded homogeneous sets $B_{n}$ and bounded homogeneous maps $\varphi_{n}: X \rightarrow X$ such that the sequence $T \circ \phi_{B_{n}} \circ \varphi_{n}$ converges to $T$ in the norm.

(v) $T$ is essentially compact and there are bounded homogeneous maps $\varphi_{n}$ : $X \rightarrow X$ and homogeneous maps $\varphi_{n}^{p}$ that are strongly dominated by $\phi_{p}$ such that the sequence $T \circ \varphi_{n}^{p} \circ \varphi_{n}$ converges to $T$ in the norm. 
Proof. (i) $\Rightarrow$ (ii). If the operator is compact, then following the ideas in the proof of Theorem 3, for each $n$ we find finitely many functions $g_{1}, \ldots, g_{l} \in X_{\left[1 / p^{\prime}\right]}$ and $h_{1}, \ldots, h_{l} \in B_{[1 / p]}$ such that

$$
T\left(B_{X}\right) \subset \bigcup_{i=1}^{l} T\left(g_{i} h_{i}\right)+\frac{1}{n} B_{E} .
$$

Define $\mathbf{g}_{n}:=\sum_{i=1}^{l}\left|g_{i}\right| \in X_{\left[1 / p^{\prime}\right]}$. Take $k_{i} \in B_{L^{\infty}(\mu)}$ such that $h_{i} g_{i}=k_{i}\left|h_{i}\right| \mathbf{g}_{n}$. By a disjointification argument, we can find disjoint sets $C_{i} \subset T\left(h_{i} g_{i}\right)+\frac{1}{n} B_{E}$ such that $\bigcup_{i=1}^{l} T\left(g_{i} h_{i}\right)+\frac{1}{n} B_{E}=\bigcup_{i=1}^{l} C_{i}+\frac{1}{n} B_{E}$. Define $\varphi_{n}: X \rightarrow X_{[1 / p]}$ given by $\varphi_{n}(f)=k_{i}\left|h_{i}\right|\|f\|$ if $\frac{f}{\|f\|} \in T^{-1}\left(C_{i}+\frac{1}{n} B_{E}\right)$ and consider $\mathbf{h}_{n}=\sum_{i=1}^{l}\left|h_{i}\right| \in X_{[1 / p]}$. For any $f \in B_{X},\left|\varphi_{n}(f)\right| \leq \mathbf{h}_{\mathbf{n}}$ and then $\varphi_{n}\left(B_{X}\right)$ is order bounded. Moreover, for $f \in B_{X}$,

$$
\left\|T(f)-T\left(\varphi_{n}(f) \mathbf{g}_{n}\right)\right\| \leq \frac{1}{n}
$$

and then the sequence $T \circ\left(\mathrm{g}_{n} \cdot \varphi_{n}\right)$ converges to $T$ in norm.

(ii) $\Rightarrow$ (iii) Take as new $\varphi_{n}$ the map $\mathbf{g}_{n} \cdot \varphi_{n}$ above.

(iii) $\Rightarrow$ (iv) is clear by taking for $B_{n}$ the homogeneous hull of $\varphi_{n}\left(B_{X}\right)$.

(iv) $\Rightarrow$ (v) is a consequence of Proposition 2 with $\varphi_{n}^{p}=\phi_{B_{n}}$.

(v) $\Rightarrow$ (i) Let $\epsilon>0$ and take $n$ such that $T(f)-T \circ \varphi_{n}^{p} \circ \varphi_{n}(f) \in \epsilon B_{E}$ for every $f \in B_{X}$. Since $\varphi_{n}\left(B_{X}\right)$ is bounded and $\varphi_{n}^{p}$ is strongly dominated by $\phi_{p}$, by Proposition $2 \varphi_{n}^{p}\left(\varphi_{n}\left(B_{X}\right)\right)$ is uniformly $\mu$-absolutely continuous. The essentially compactness of $T$ yields that $T\left(\varphi_{n}^{p}\left(\varphi_{n}\left(B_{X}\right)\right)\right)$ is relatively compact. By Grothendieck characterization of compactness we conclude that $T\left(B_{X}\right)$ is relatively compact.

Just changing the $T$ in the approximating homogeneous maps in the above theorem by sequences of bounded homogeneous maps $R_{n}$ we get, among others, the following characterization.

Let $E$ be a Banach space, $X(\mu)$ be an order continuous Banach function space with the Fatou property and $1<p<\infty$. The following statements for a linear operator $T: X(\mu) \rightarrow E$ are equivalent.

(i) $T$ is compact.

(ii) $T$ is essentially compact and there are order bounded homogeneous sets $B_{n}$, bounded homogeneous maps $\varphi_{n}: X \rightarrow X$ and uniformly $\mu$-absolutely continuous homogeneous maps $R_{n}: X \rightarrow E$ such that the sequence $R_{n}$ 。 $\phi_{B_{n}} \circ \varphi_{n}$ converges to $T$ in the norm.

Notice that the characterization of compactness given in the above result can be written also in terms of inequalities: an operator $T$ is compact if and only if it is essentially compact and for each $n \in \mathbb{N}$ there are homogeneous maps $\varphi_{n}, \varphi_{n}^{p}: X \rightarrow$ $X$ such that for $f_{1}, \ldots, f_{n} \in X$ and $A_{1}, \ldots, A_{n} \in \Sigma$,

$$
\left\|\sum_{i=1}^{n}\left|\varphi_{n}^{p} \circ \varphi_{n}\left(f_{i}\right)\right| \chi_{A_{i}}\right\|_{L^{1}(\mu)} \leq K_{n}\left\|\sum_{i=1}^{n}\left|\varphi_{n}\left(f_{i}\right)\right|^{1 / p}\right\| \varphi_{n}\left(f_{i}\right)\left\|^{1 / p^{\prime}} \chi_{A_{i}}\right\|_{\left(X_{\left[1 / p^{\prime}\right]}\right)^{\prime}} .
$$

such that $T \circ \varphi_{n}^{p} \circ \varphi_{n}$ converges to $T$ in the norm. This is a consequence of Theorem 2 and Theorem $3(\mathrm{v})$. 
Let us finish this section by showing some particular factorizations of operators through the relevant $L^{p}$-spaces. We will use a variant of the classical MaureyRosenthal Theorem due to Defant [2, Corollary 2] on factorization of homogeneous operators between Banach function spaces.

Let $E$ be a Banach space, $X(\mu)$ be an order continuous $q$-concave Banach function space, for some $1<q<\infty$, and $T: X \rightarrow E$ be a linear operator. Then the following assertions are equivalent for any $p \geq q$ :

(i) $T$ is compact.

(ii) $T$ is essentially compact and there are order bounded sets $C_{n} \subset L^{p}(\mu)$, bounded homogeneous maps $\psi_{n}: X \rightarrow L^{p}(\mu)$ satisfying $\psi_{n}\left(B_{X}\right) \subset C_{n}$ and functions $h_{n} \in L^{p}(\mu)^{X(\mu)}$ such that the sequence $\left(T \circ\left(h_{n} \cdot \psi_{n}\right)\right)_{n}$ converges to $T$ in norm.

(iii) There are order bounded sets $C_{n} \subset L^{p}(\mu)$, bounded homogeneous maps $\psi_{n}: X \rightarrow L^{p}(\mu)$ satisfying $\psi_{n}\left(B_{X}\right) \subset C_{n}$, functions $h_{n} \in L^{p}(\mu)^{X(\mu)}$ and essentially compact linear operators $T_{n}: X \rightarrow E$ such that the sequence $\left(T_{n} \circ\left(h_{n} \cdot \psi_{n}\right)\right)_{n}$ converges to $T$ in norm.

Proof. (i) $\Rightarrow$ (ii). By Theorem 3(ii) there are $g_{n} \in X_{\left[1 / p^{\prime}\right]}$, order bounded homogeneous sets $B_{n}$ and bounded homogeneous maps $\phi_{n}: X \rightarrow X_{[1 / p]}$ such that $\phi_{n}\left(B_{X}\right) \subset B_{n}$ and the sequence $T \circ\left(g_{n} \cdot \phi_{n}\right)$ converges to $T$ in the norm. Since the space $X_{[1 / p]}$ is always $p$-convex and $X$ is $p$-concave, an application of the abstract version of the Maurey-Rosenthal Theorem [2, Corollary 2] to the linear operator $M_{g_{n}}: X_{[1 / p]} \rightarrow X, M_{g_{n}}(f):=g_{n} f$, provides a function $h_{n} \in L^{p}(\mu)^{X(\mu)}$ such that the following diagram commutes:

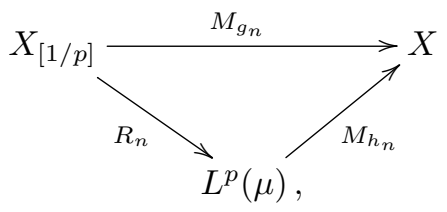

where $R_{n}$ is given by $R_{n}:=\frac{M_{g_{n}}}{h_{n}}$. Now define $C_{n}:=R_{n}\left(B_{n}\right)$ and $\psi_{n}:=R_{n} \circ \phi_{n}$.

(ii) $\Rightarrow$ (iii) is trivial.

(iii) $\Rightarrow$ (i) Let $\epsilon>0$ and take $n$ such that $T(f)-T_{n} \circ\left(h_{n} \cdot \psi_{n}\right)(f) \in \epsilon B_{E}$ for every $f \in B_{X}$. It follows from the fact that $M_{h_{n}}\left(C_{n}\right)$ is order bounded and its image by an essentially compact operator is relatively compact that the set $T_{n} \circ\left(h_{n} \cdot \psi_{n}\right)\left(B_{X}\right)$ is relatively compact. The well-known Grothendieck's characterization of compact sets yields that $T\left(B_{X}\right)$ is relatively compact.

Notice that in the case that the space $L^{p}(\mu)^{X(\mu)}$ is order continuous then the assertions in Corollary 3 are equivalent to the following one:

iv) There are bounded homogeneous maps $\psi_{n}: X(\mu) \rightarrow L^{p}(\mu)$, functions $h_{n} \in$ $L^{p}(\mu)^{X(\mu)}$ and essentially compact linear operators $T_{n}: X \rightarrow E$ such that the sequence $\left(T_{n} \circ\left(h_{n} \cdot \psi_{n}\right)\right)_{n}$ converges to $T$ in norm.

This is so because we do not need to ask for order boundedness to the sets $\psi_{n}\left(B_{X}\right)$, since then the sets $h_{n} \cdot \psi_{n}\left(B_{X}\right)$ are uniformly $\mu$-absolutely continuous and so the composition of $h_{n} \cdot \psi_{n}$ with an essentially compact operator gives a compact homogeneous map. 


\section{Applications: Compact optimal extensions for essentially COMPaCt OPERATORS}

If $X(\mu)$ is an order continuous Banach function space over a finite measure $\mu$ then any continuous $\mu$-determined linear operator $T$ from $X(\mu)$ into a Banach space $E$ can be extended continuously to $L^{1}\left(m_{T}\right)$, where $m_{T}$ is the vector measure associated to $T$. This space is the optimal domain for $T$, i.e. the biggest order continuous space to which $T$ can be extended (see [12, Theorem 4.14] and the references therein). In that case, the optimal extension of $T$ is given by the associated integration operator $I_{m_{T}}: L^{1}\left(m_{T}\right) \rightarrow E$.

In this section we study the following problem: Let $X(\mu)$ be an order continuous Banach function space over a finite measure $\mu$ and let $T: X(\mu) \rightarrow E$ be a Banach space valued operator with compact essential range. When is the maximal extension of $T$ compact?

A closely related question has been studied in [10] recently. In that paper it is shown that a $\mu$-determined compact operator $T$ allows a maximal compact extension - not necessarily to the space $L^{1}\left(m_{T}\right)$ - if and only if the associated integration map $I_{m_{T}}: L^{1}\left(m_{T}\right) \rightarrow E$ is compact (see Theorem 1.1 and Lemma 2.2 in [10] for some arguments that are close to the ones we use in the proof of Theorem 4). Moreover, it is well known that compactness of the integration map of a vector measure $m$ imply that $L^{1}(m)=L^{1}(|m|)$ (see [11] or Proposition 3.48 in [12] and the references therein).

The following reformulation of Theorem 3 for the integration operator $I_{m}$ associated to a vector measure $m$ provides the main result of this section. Recall that $\mathcal{R}(m)$ is the range of $m$.

Let $1 \leq p<\infty$. The following statements are equivalent.

(i) The integration operator $I_{m}: L^{1}(m) \rightarrow\left(E,\|\|_{E}\right)$ is compact.

(ii) $\mathcal{R}(m)$ is relatively compact and for every $\varepsilon>0$ there exists $g_{\varepsilon} \in L^{p^{\prime}}(m)$ such that $I_{m}\left(B_{L^{1}(m)}\right) \subset I_{m}\left(g_{\varepsilon} B_{L^{p}(m)}\right)+\varepsilon B_{E}$.

(iii) $\mathcal{R}(m)$ is relatively compact and for every $\varepsilon>0$ there exists $K_{\varepsilon}>0$ such that $I_{m}\left(B_{L^{1}(m)}\right) \subset I_{m}\left(K_{\varepsilon} B_{L^{p}(m)}\right)+\varepsilon B_{E}$.

Let $1<p<\infty$, let $X(\mu)$ be an order continuous Banach function space, $E$ a Banach space and $T: X(\mu) \rightarrow E$ a $\mu$-determined continuous linear operator. The following statements are equivalent:

(i) The optimal domain of $T$ is $L^{1}\left(\left|m_{T}\right|\right)$ and the extension $I_{m_{T}}$ is compact, i.e. $T$ factorizes compactly as

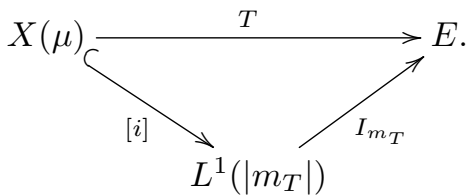

(ii) $T$ is essentially compact, and for each $\varepsilon>0$ there is a function $h_{\varepsilon} \in X_{\left[1 / p^{\prime}\right]}$ and a bounded homogeneous map $\phi_{\varepsilon}: X \rightarrow X_{[1 / p]}$ such that

$$
\sup _{A \in \Sigma}\left\|T\left(\phi_{\varepsilon}(f)^{p} \chi_{A}\right)\right\|^{1 / p} \leq \sup _{A \in \Sigma}\left\|T\left(f \chi_{A}\right)\right\|
$$

and

$$
\left\|T\left(f-h_{\varepsilon} \phi_{\varepsilon}(f)\right)\right\|<\varepsilon \cdot \sup _{A \in \Sigma}\left\|T\left(f \chi_{A}\right)\right\|, \quad f \in X .
$$


(iii) $T$ is essentially compact and for each $n \in \mathbb{N}$ there is a constant $k_{n}>0$ and a bounded homogeneous map $\phi_{n}: X \rightarrow X_{[1 / p]}$ such that for all $f \in X$,

$$
\begin{gathered}
\max \left\{k_{n} \cdot \sup _{A \in \Sigma}\left\|T\left(\phi_{n}(f)^{p} \chi_{A}\right)\right\|^{1 / p}, n \cdot\left\|T\left(f-\phi_{n}(f)\right)\right\|\right\} \\
\leq \sup _{A \in \Sigma}\left\|T\left(f \chi_{A}\right)\right\| .
\end{gathered}
$$

Proof. Let us start the proof by establishing that, taking apart the essential compactness assumption, (ii) is equivalent to the fact that for each $\varepsilon>0$ there is $h_{\varepsilon} \in X_{\left[1 / p^{\prime}\right]}$ such that

$$
\sup _{f \in B_{L^{1}\left(m_{T}\right)} \cap X(\mu)}\left(\inf _{g \in B_{L^{p}\left(m_{T}\right)} \cap X_{[1 / p]}}\left(\left\|T\left(f-h_{\varepsilon} g\right)\right\|\right)\right)<\varepsilon .
$$

To see this, just note that the computation of the infimum in this statement can be used to define a function $\phi_{\varepsilon}: B_{L^{1}\left(m_{T}\right)} \cap X \rightarrow B_{L^{p}\left(m_{T}\right)} \cap X_{[1 / p]}$ that carries each function to one that makes the inequality less than $\varepsilon$. In fact, it can be assumed to be a bounded homogeneous function $\left(X,\|\cdot\|_{L^{1}\left(m_{T}\right)}\right) \rightarrow\left(X_{[1 / p]},\|\cdot\|_{L^{p}\left(m_{T}\right)}\right)$ just by taking $\phi_{\varepsilon}^{\prime}(f)=\|f\|_{L^{1}\left(m_{T}\right)} \phi_{\varepsilon}\left(f /\|f\|_{L^{1}\left(m_{T}\right)}\right), f \in X$. This shows that the condition above is equivalent to the existence for each $\varepsilon>0$ of a function $h_{\varepsilon} \in X_{\left[1 / p^{\prime}\right]}$ such that

$$
\left\|T\left(f-h_{\varepsilon} \phi_{\varepsilon}^{\prime}(f)\right)\right\|<\varepsilon \sup _{A \in \Sigma}\left\|T\left(f \chi_{A}\right)\right\|, \quad f \in X .
$$

Recall that the expression $\sup _{A \in \Sigma}\left\|T\left(f \chi_{A}\right)\right\|$ is equivalent to $\|f\|_{L^{1}\left(m_{T}\right)}$ for functions belonging to $X$. The converse argument follows too, and so we have the equivalence.

Exactly in the same way, it can be proved, taking again apart the essential compactness assumption, that (iii) is equivalent to the fact that for each $\varepsilon>0$ there is a constant $K_{\varepsilon}>0$ such that

$$
\sup _{f \in B_{L^{1}\left(m_{T}\right)} \cap X(\mu)}\left(\inf _{g \in B_{L^{p}\left(m_{T}\right)} \cap X_{[1 / p]}}\left(\left\|T\left(f-K_{\varepsilon} g\right)\right\|\right)\right)<\varepsilon
$$

holds (as in (2) but with a constant $K_{\varepsilon}$ instead of a function $h_{\varepsilon}$ ), just using the fact that this relation is satisfied for $\varepsilon=1 / n$ and $K_{\varepsilon}=1 / k_{n}$.

We start proving $(\mathrm{iii}) \Rightarrow(\mathrm{i})$. Let us see that under the assumption of the property given by (3), the inclusions appearing in Proposition 4(iii) hold. Indeed, let $\varepsilon>0$ and $f \in B_{L^{1}\left(m_{T}\right)}$. Since the simple functions are dense in $L^{1}\left(m_{T}\right)$, there exists a simple function $s \in B_{L^{1}\left(m_{T}\right)}$ such that

$$
\|f-s\|_{L^{1}\left(m_{T}\right)}<\frac{\varepsilon}{2\left(\left\|I_{m_{T}}\right\|+1\right)} .
$$

As $s \in B_{L^{1}\left(m_{T}\right)} \cap X(\mu)$ we can apply part (iii) — actually we apply its equivalent formulation (3) - to $s$ : there exist $K_{\varepsilon / 2}>0$ and $g \in B_{L^{p}\left(m_{T}\right)} \cap X_{[1 / p]}$ such that $\left\|T\left(s-K_{\varepsilon / 2} g\right)\right\|<\varepsilon / 2$. Then, by (4) we get

$$
\begin{aligned}
\left\|I_{m_{T}}\left(f-K_{\varepsilon / 2} g\right)\right\| & \leq\left\|I_{m_{T}}(f-s)+T\left(s-K_{\varepsilon / 2} g\right)\right\| \\
& \leq\left\|I_{m_{T}}\right\| \frac{\varepsilon}{2\left(\left\|I_{m_{T}}\right\|+1\right)}+\frac{\varepsilon}{2}<\varepsilon .
\end{aligned}
$$


Then $I_{m_{T}}$ satisfies the inclusion property in Proposition 4(iii). On the other hand, the essential compactness of $T$ is trivially equivalent to $m_{T}$ having relatively compact range. Proposition 4 applied to $m_{T}$ yields that $I_{m_{T}}$ is compact. By [11, Theorems 1 and 4] $L^{1}\left(m_{T}\right)=L^{1}\left(\left|m_{T}\right|\right)$ and then, since $L^{1}\left(m_{T}\right)$ is the optimal domain of the map, we obtain the desired factorization diagram for $T$ through $L^{1}\left(\left|m_{T}\right|\right)$.

The proof of (ii) $\Rightarrow(\mathrm{i})$ is similar using (2) and Proposition 4(ii) instead of (3) and Proposition 4(iii) respectively.

We finally prove (i) $\Rightarrow$ (iii) (the implication (i) $\Rightarrow$ (iii) is similar). Since $I_{m_{T}}$ is compact, $T$ is compact and so essentially compact. Compactness of $I_{m_{T}}$ gives also by Proposition 4 that for each $\varepsilon>0$ we find a constant $K_{\varepsilon}>0$ such that $I_{m_{T}}\left(B_{L^{1}\left(m_{T}\right)}\right) \subseteq I_{m_{T}}\left(K_{\varepsilon} B_{L^{p}\left(m_{T}\right)}\right)+\varepsilon B_{E}$. Taking into account that $X_{[1 / p]}$ is dense in $L^{p}\left(m_{T}\right),(3)$ and so (iii) follow easily.

More results can be obtained on compact optimal extensions by applying the outcomes of Section 3. For example, the following result is a direct application of Corollary 3 . We need that $L^{p}\left(m_{T}\right)=L_{w}^{p}\left(m_{T}\right)$ for assuring the Fatou property for $L^{p}\left(m_{T}\right)$, what happens for instance if $E$ is reflexive (see [5] for sufficient conditions for this to hold).

Let $1<p<\infty$ and let $X(\mu)$ be an order continuous Banach function space. Let $T: X(\mu) \rightarrow E$ be an essentially compact operator and $\nu$ a Rybakov measure for $m_{T}$. Suppose that $L^{p}\left(m_{T}\right)=L_{w}^{p}\left(m_{T}\right)$ and there is an increasing sequence of solid homogeneous sets $\left\{B_{n}\right\}_{n=1}^{\infty}$ in $X$ satisfying that for each $n \in \mathbb{N}$ there is a constant $K_{n}>0$ such that for each finite set $f_{1}, \ldots, f_{k} \in B_{n}$,

$$
\left\|\sum_{i=1}^{k}\left|f_{i}\right|\right\|_{L^{1}(\nu)} \leq K_{n} \cdot\left\|\sum_{i=1}^{k}\left|f_{i}\right|^{1 / p}\right\| f_{i}\left\|^{1 / p^{\prime}}\right\|_{\left(L^{p^{\prime}}\left(m_{T}\right)\right)^{\prime}},
$$

and $T \circ \phi_{B_{n}} \rightarrow T$ in the operator norm. Then $I_{m_{T}}$ is compact.

The first author gratefully acknowledges the support of the Ministerio de Economía y Competitividad (Spain), under project \#MTM2011-22417. The second author gratefully acknowledges the support of the Ministerio de Economía y Competitividad (Spain), under project \#MTM2012-36740-c02-02.

\section{REFERENCES}

[1] J. M. Calabuig, O. Delgado and E. A. Sánchez Pérez, Factorizing operators on Banach function spaces through spaces of multiplication operators, J. Math. Anal. Appl. 364, 88-103 (2010)

[2] A. Defant, Variants of the Maurey-Rosenthal theorem for quasi Köthe function spaces, Positivity 5 153-175 (2001)

[3] O. Delgado and E.A. Sánchez Pérez, Strong factorizations between couples of operators on Banach spaces, J. Convex Anal. To appear.

[4] J. Diestel and J. J. Uhl, Vector Measures, Math. Surveys, vol. 15, Amer. Math. Soc., Providence, RI (1977)

[5] A. Fernández, F. Mayoral, F. Naranjo, C. Sáez and E.A. Sánchez-Pérez, Spaces of p-integrable functions with respect to a vector measure, Positivity 10, 1-16 (2006)

[6] I. Ferrando and J. Rodríguez, The weak topology on $L_{p}$ of a vector measure, Topology Appl. 155(13), 1439-1444 (2008)

[7] J. Lindenstrauss and L. Tzafriri, Classical Banach Spaces, II, Springer, Berlin (1996)

[8] L. Maligranda and L. E. Persson, Generalized duality of some Banach function spaces, Indag. Math. 51, 323-338 (1989) 
[9] P. Meyer-Nieberg, Banach lattices, Springer, Berlin (1991)

[10] S. Okada, Does a compact operator admit a maximal domain for its compact linear extension?, in: Vector Measures, Integration and Related Topics, Operator Theory. Advances and Applications, 201, pp.313-322. Birkhäuser, Basel (2009)

[11] S. Okada, W.J. Ricker, and L. Rodríguez-Piazza, Compactness of the integration operator associated with a vector measure, Studia Math. 150(2), 133-149 (2002)

[12] S. Okada, W.J. Ricker and E.A. Sánchez Pérez, Optimal domain and integral extension of operators acting in function spaces. Operator Theory: Advances and Applications, 180. Birkhäuser, Basel (2008)

[13] E. A. Sánchez Pérez, Compactness arguments for spaces of p-integrable functions with respect to a vector measure and factorization of operators through Lebesgue-Bochner spaces, Illinois J. Math. 45 3, 907-923 (2001)

Pilar Rueda

Departamento de Análisis Matemático, Universidad de Valencia, 46100 Burjassot Valencia, Spain.

pilar.rueda@uv.es

Enrique A. Sánchez-Pérez

Instituto Universitario de Matemática Pura y Aplicada, Universitat Politècnica de Valencia, Camino de Vera s/n, 46022 Valencia, Spain.

Tel.: +34-963877000

Fax: +34-963877669

easancpe@mat.upv.es 